\title{
Quantitative SIMS analysis of GSJ rock reference samples
}

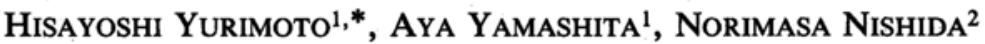 \\ and SHIGeHo SuENo ${ }^{1}$ \\ Institute of Geoscience ${ }^{1}$, \\ and Chemical Analysis Center ${ }^{2}$, The University of Tsukuba, \\ Tsukuba, Ibaraki, 305 Japan
}

(Received November 1, 1989; Accepted January 6, 1990)

\begin{abstract}
Quantitative micro-analysis of silicate glasses has been developed using secondary ion mass spectrometry (SIMS). The standard rock references, issued by the Geological Survey of Japan (GSJ), were fused into glass beads and used as standards for SIMS analysis. Thirty eight elements at major, minor and trace concentration levels, were analyzed simultaneously on the standard glasses using an energyfiltering technique. In the concentration range of the glass standards, linear relationships were established for the following elements: $\mathrm{Li}, \mathrm{Be}, \mathrm{B}, \mathrm{Na}, \mathrm{Mg}, \mathrm{Al}, \mathrm{P}, \mathrm{K}, \mathrm{Ca}, \mathrm{Sc}, \mathrm{Ti}, \mathrm{V}, \mathrm{Cr}, \mathrm{Mn}, \mathrm{Fe}, \mathrm{Co}, \mathrm{Cu}, \mathrm{Rb}, \mathrm{Sr}$, $\mathrm{Y}, \mathrm{Zr}, \mathrm{Cs}, \mathrm{Ba}, \mathrm{La}, \mathrm{Ce}, \mathrm{Pr}, \mathrm{Nd}, \mathrm{Sm}, \mathrm{Eu}, \mathrm{Gd}, \mathrm{Tb}, \mathrm{Dy}, \mathrm{Ho}, \mathrm{Er}, \mathrm{Tm}, \mathrm{Yb}, \mathrm{Lu}$ and Hf. The typical accuracy of the analyses are within $10 \%$ of recommended values of GSJ standards for the elements over $1 \mathrm{ppm}$.
\end{abstract}

\section{INTRODUCTION}

Secondary ion mass spectrometry (SIMS) has attracted the interest of geochemists for over a decade because it offers the potential of complete in situ chemical and isotopic analyses of solid materials with ppm to ppb sensitivity and micron-scale spatial resolution. Several investigators have applied SIMS to the quantitative elemental analysis of minerals. However, progress of quantitative SIMS has been limited and not widely accepted by earth scientists.

The presence of molecular and multiply charged ions in the secondary ion mass spectrum of geological samples is a serious source of major complications, which is inherent in SIMS. Molecular interferences can be minimized by high mass resolution (Steele et al., 1981; Yurimoto and Sueno, 1984; Reed, 1984) or energy filtering (Shimizu et al., 1978; Ray and Hart, 1982; Metson et al., 1983; Nesbitt et al., 1986; Zinner and Crozaz, 1986). Both methods have distinct advantages. For example, energy filtering is efficient in eliminating molecular ion interferences for masses greater than $\sim 60$ (Shimi$\mathrm{zu}$ and Hart, 1982) and SIMS adjustment and peak jumping among masses are easier, while the high mass resolution mode is effective for resolving isobars, multiply charged ions and certain types of molecular ions (e.g. hydride).

The existence of matrix effects is another serious source of major complications in SIMS. SIMS quantification can be pursued using either theoretical or empirical approaches. Theoretical approaches, however, are currently impractical due to the uncertainties of compositional matrix effects on secondary-ion yields (Williams, 1985). The most successful method involves the use of standards to obtain relative sensitivity factors of the measured elements (Shimizu et al., 1978; Steele et al., 1981; Ray and Hart, 1982; Reed, 1984, 1986; Yurimoto and Sueno, 1985; Zinner and Crozaz, 1986). Major problems with this technique are the difficulty of obtaining suitable

*Present Address: Institute for the Study of Mineral Deposits, South Dakota School of Mines and Technology, Rapid City, South Dakota 57701, U.S.A. 
standards and the large numbers of standards required.

We have developed analytical methods for quantitative SIMS (Yurimoto and Sueno, 1984, 1985 and 1987; Yurimoto et al., 1989). In this paper, we provide instrumental and standardization details for trace element analysis of silicates by SIMS. We also examine systematically the contribution of molecular-ion interferences and matrix effects to the secondary ion mass spectra of ten silicate-glass standards. An empirical approach based on energy filtering was used to establish calibration curves for 38 elements in glasses prepared from rock reference standards.

\section{Analytical Methods}

Powdered standard rock samples (about $1 \mathrm{~g}$ each of JA-1, JA-2, JA-3, JB-1, JB-1a, JB-2, JB-3, JF-1, JF-2, JG-1, JG-1a, JG-2, JG-3, JGb1, JP-1, JR-1 and JR-2), issued by the Geological Survey of Japan (GSJ), were loaded into $\mathrm{Pt}$ capsules and fused at $1340^{\circ} \mathrm{C}$ for 5 minutes under atmospheric condition. The melt was quenched in water to produce glass. For JR1 and JR-2, which are clear natural rhyolite glasses, the original rock specimen was also prepared. The samples were sliced by diamond saw and mounted in an epoxy pellet with final polishing by $0.3 \mu \mathrm{m}$ alumina paste.

Data were obtained on a Cameca IMS-3F secondary ion microscope at the University of Tsukuba. The primary ion beam was mass filtered ${ }^{16} \mathrm{O}^{-}$-accelerated to $14.5 \mathrm{keV}$ and adjusted for a beam current of about $60 \mathrm{nA}$ and a spot size of about $80 \mu \mathrm{m}$. The primary beam was rastered over $100 \times 100 \mu \mathrm{m}$ area during irradiation. Secondary ions sputtered were collected from the central area of $60 \mu \mathrm{m}$ in diameter using a mechanical aperture. Kinetic energy filtering was achieved by offsetting the sample accelerating voltage $(-100 \mathrm{~V})$ while keeping the setting of the electrostatic analyzer voltage and the width and position of the energy slit constant. The energy band-pass was set to accept a $20 \mathrm{eV}$ window. The exit slit was at its maximum opening resulting in a mass resolving power of $\sim 500$.
Positive secondary ion intensities were measured, typically for 10 seconds, in order of mass number using peak jumping. The sequence was repeated from 10 to 20 times depending on the count rates of each mass.

Problems caused by electrostatic charging of the sample surface under sputtering by the primary ion beam were virtually eliminated by $\sim 20 \mathrm{~nm}$ of gold film evaporated to the surface and by monitoring the kinetic energy distribution maximum of $\mathrm{Si}^{+}$.

Other analytical and instrumental conditions were similar to those employed by Yurimoto and Sueno (1984) and Yurimoto et al. (1989).

\section{Glass Standards}

Clear glass under optical microscopy has been prepared for ten kinds of the rock references (JA-1, JA-2, JA-3, JB-1, JB-1a, JB-2, JB-3, JGb-1, JR-1 and JR-2). Homogeneity of these glasses was evaluated by independent SIMS runs at more than ten different points of each glass. Results are shown in Table 1.

Coefficients for variation (CV\%), which is relative standard deviation for reproduction at different points, are $<10 \%$ for major elements and $\sim 10 \%$ for trace elements ( $>10 \mathrm{ppm}$ ). This indicates that chemical heterogeneity of the samples does not exceed $10 \%$. Since the CV\% include fluctuations of intensity ratios depending on the daily changes of instrumental conditions of SIMS as well as chemical heterogeneity in the sample, chemical heterogeneity of the standards must be much smaller, possibly less than $5 \%$ at the analyzing volume, i.e. $60 \mu \mathrm{m}$ in diameter and less than $1 \mu \mathrm{m}$ depth (<10 ng).

There were no obvious differences in intensity ratios determined for the fused glasses and the original rock specimens of JR-series standards. Therefore, it can be concluded that compositional changes during the melting process are negligible, at least for the elements observed in this study.

For JF-1, JG-1, JG-1a, JG-2 and JP-1, some unmelted crystals were observed in the fused glass with the optical microscope. Although the 

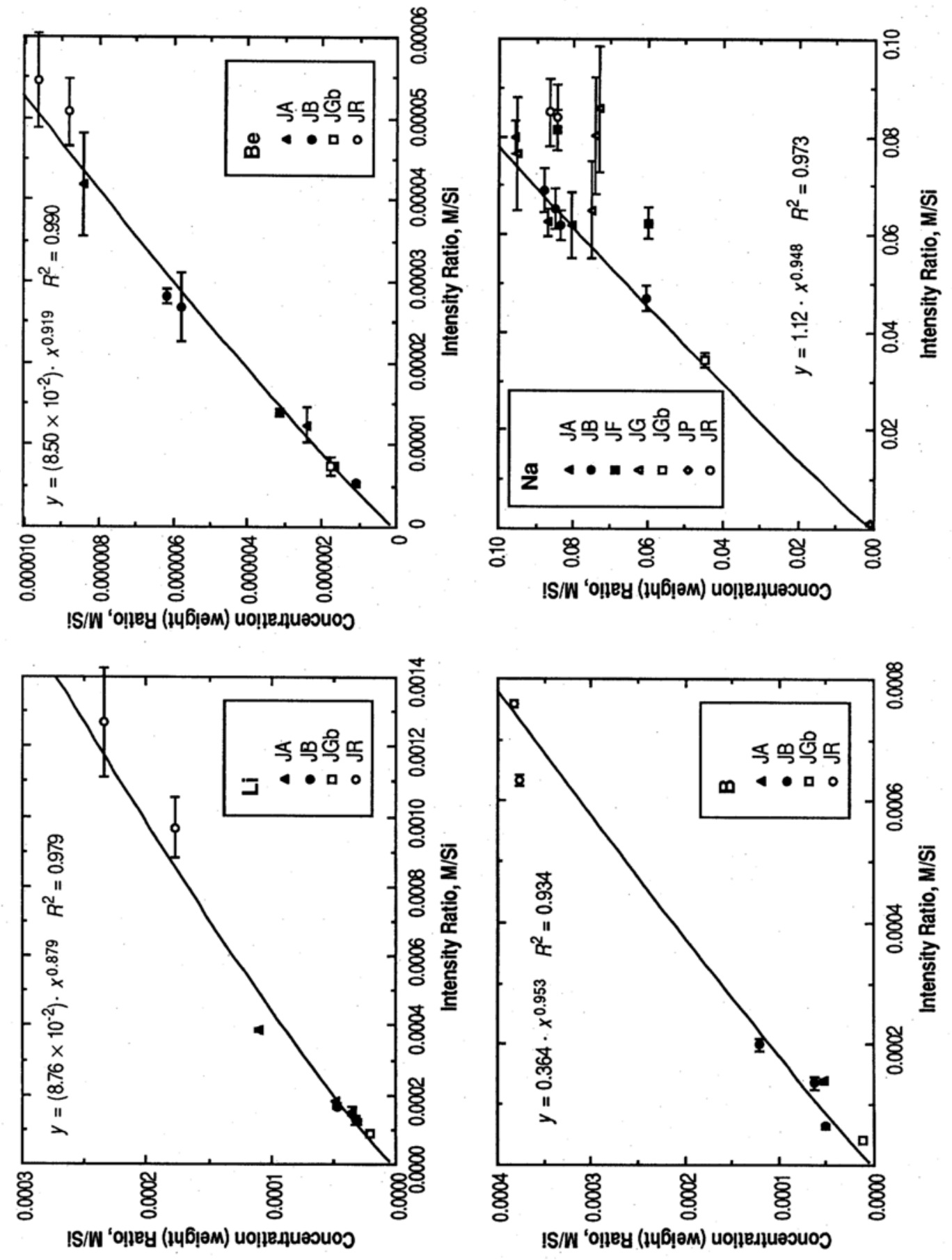


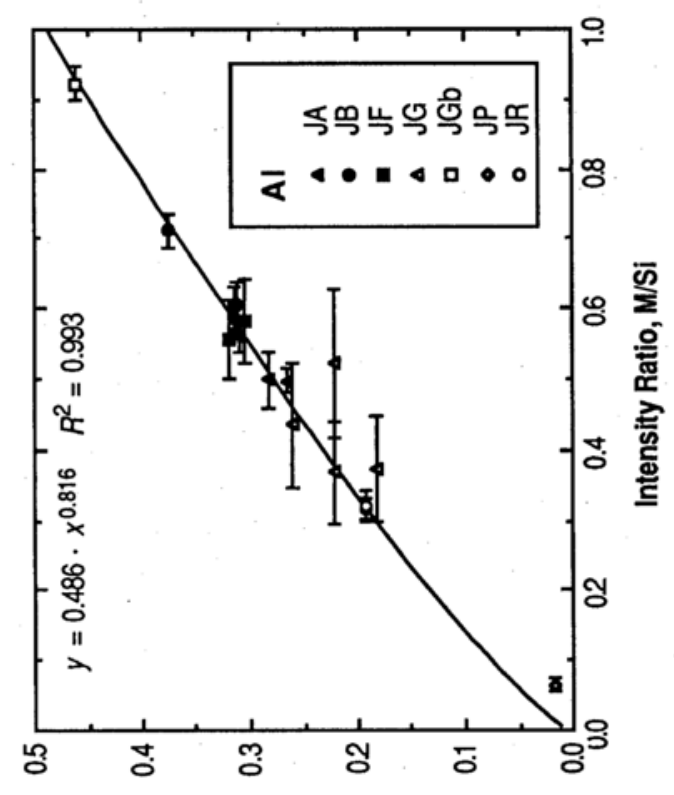

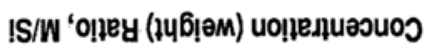

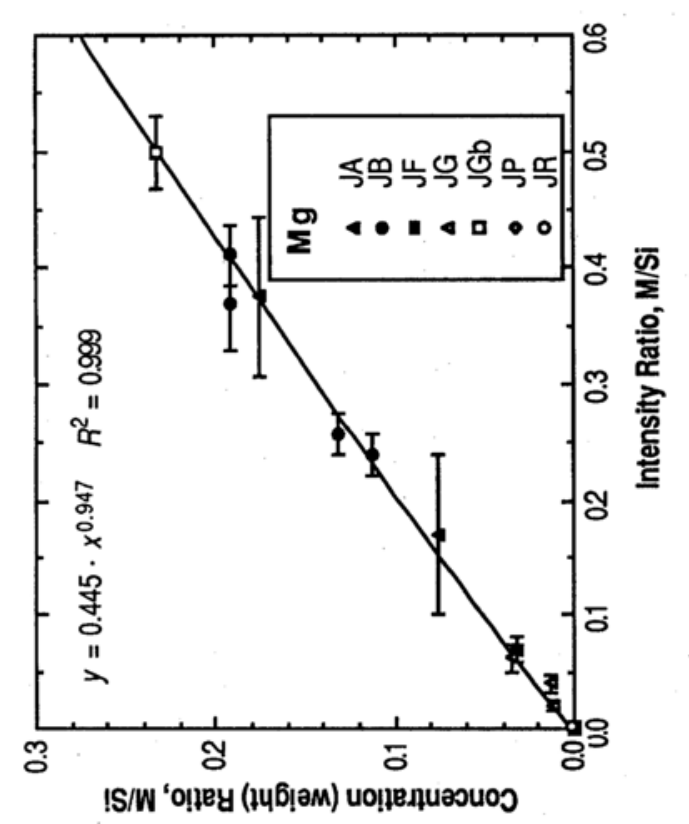

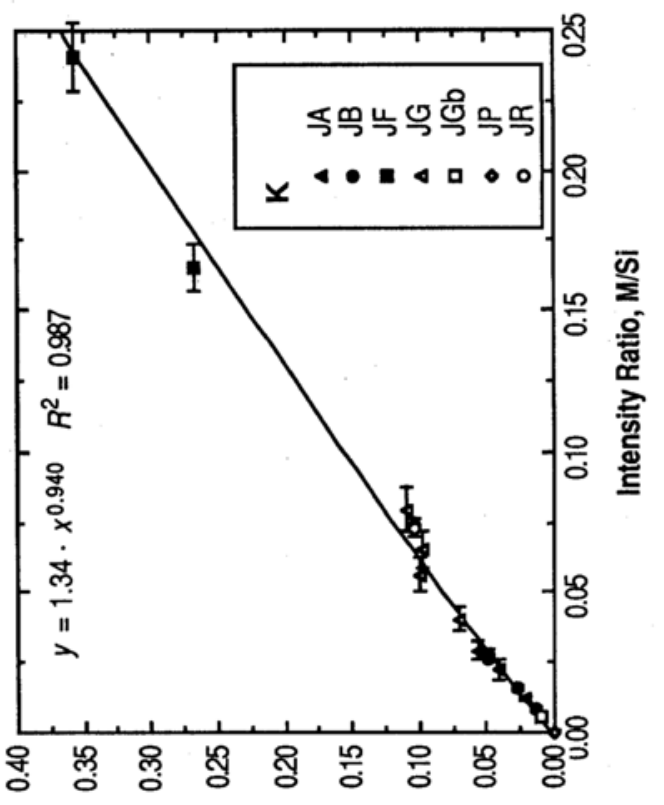

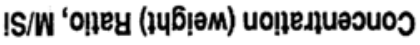

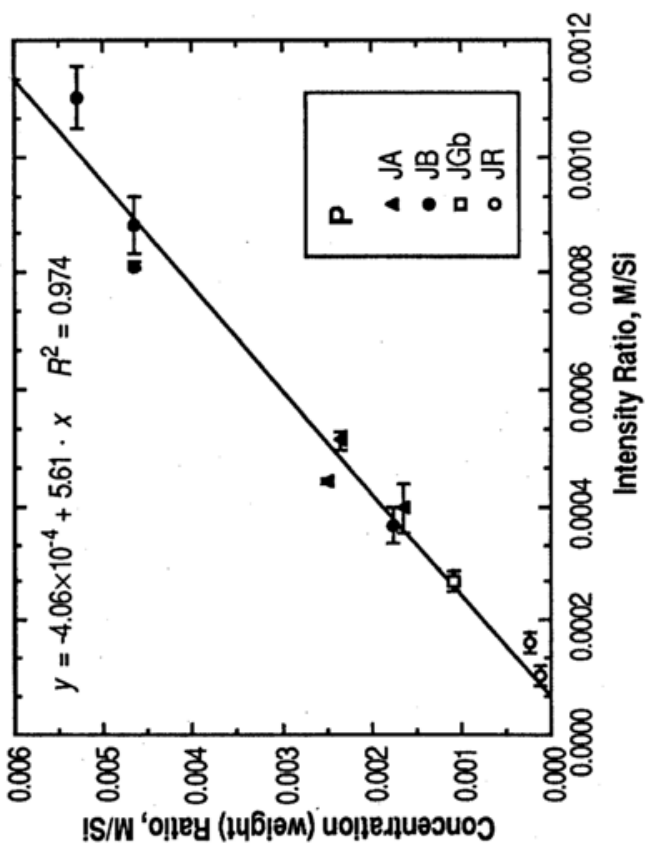




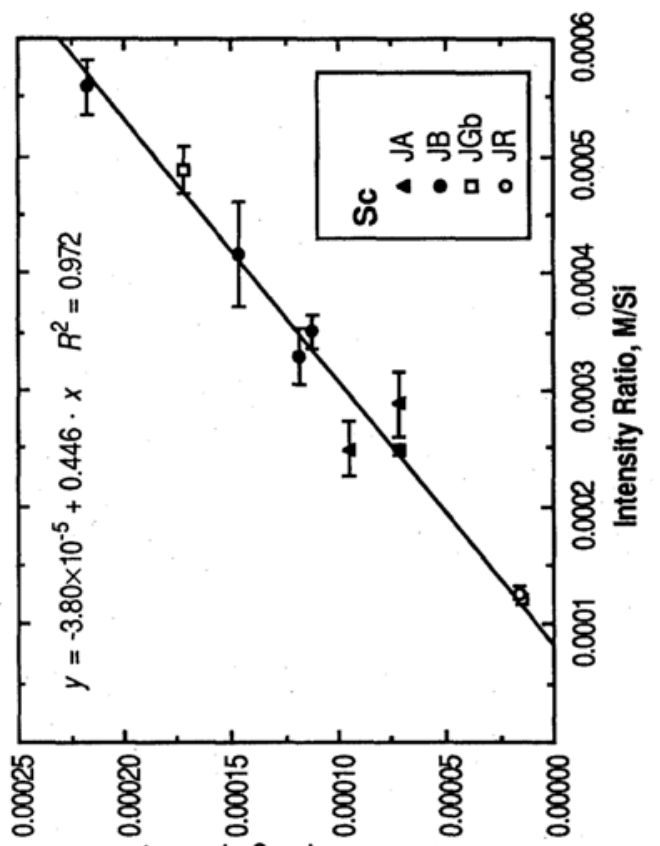

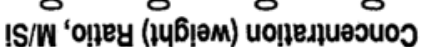

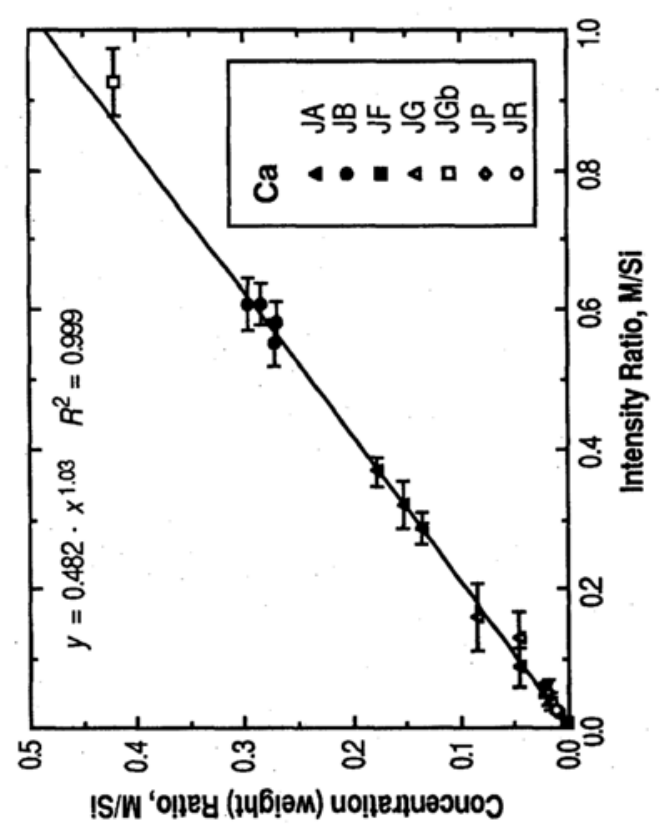

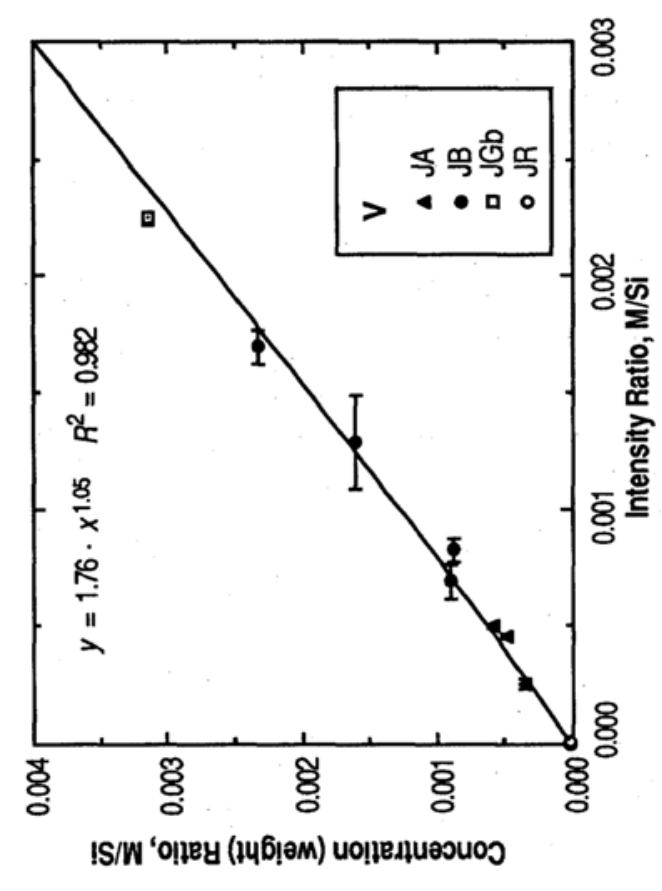

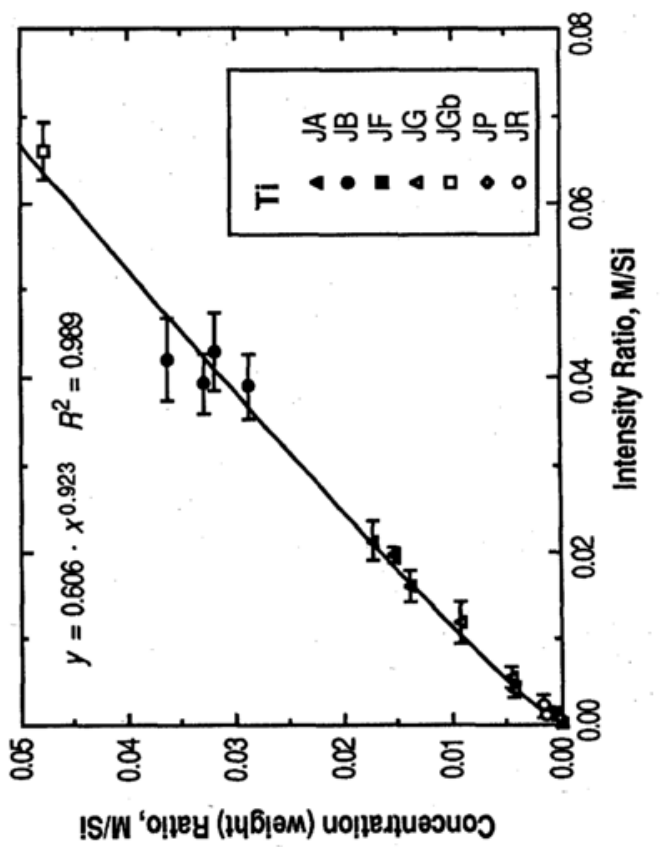




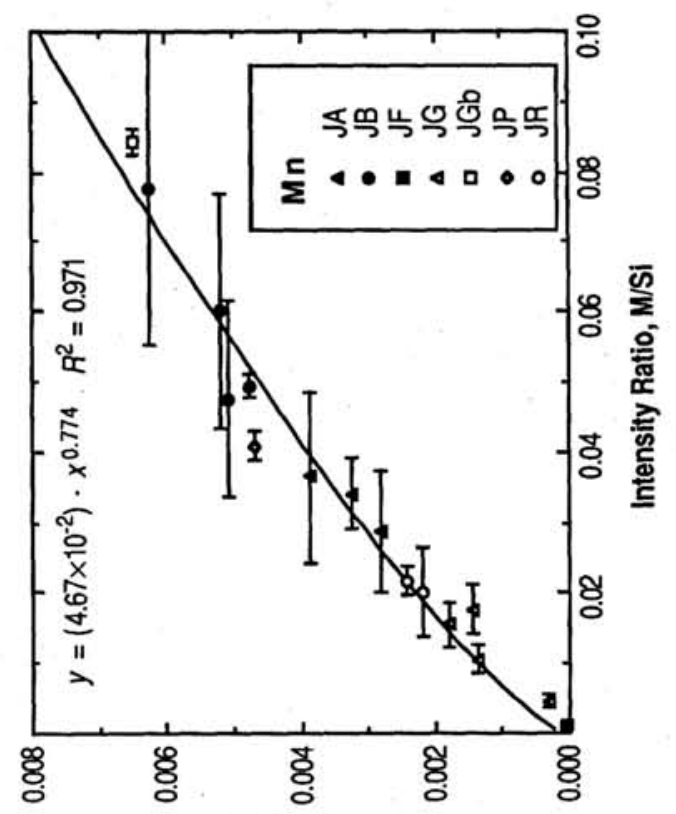

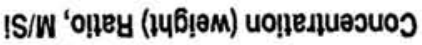

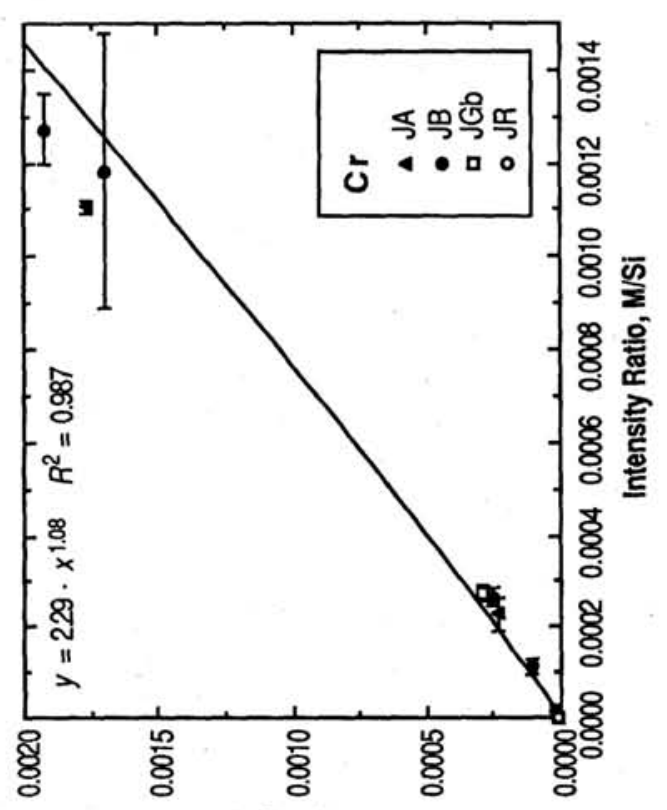

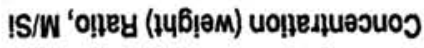
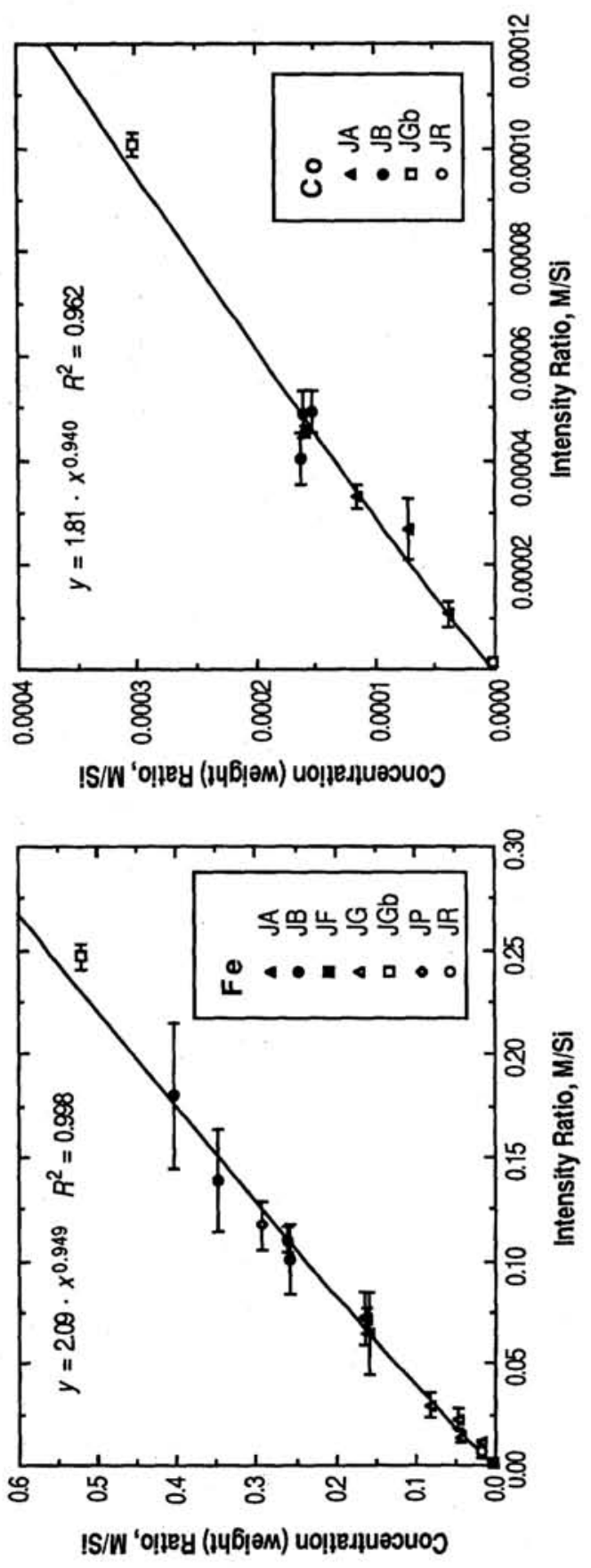


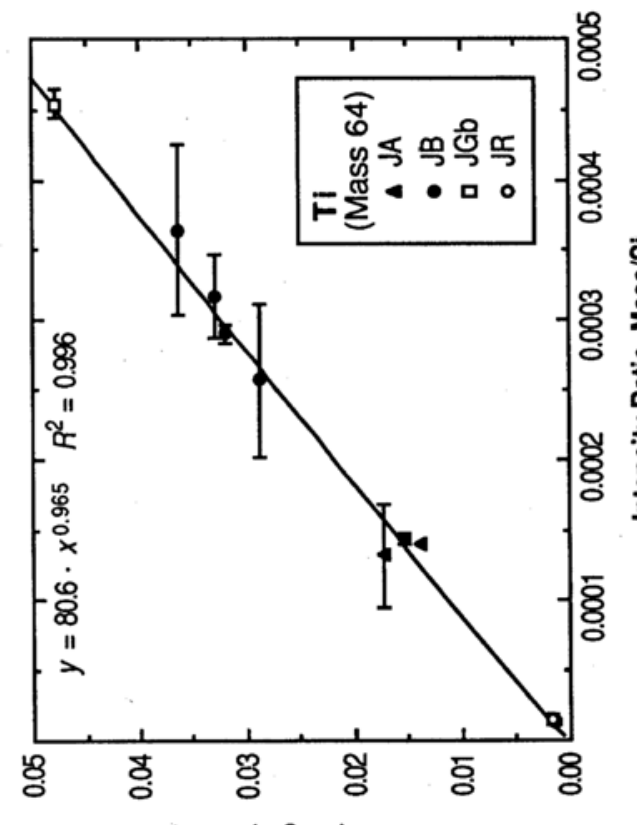

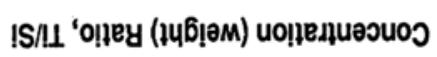

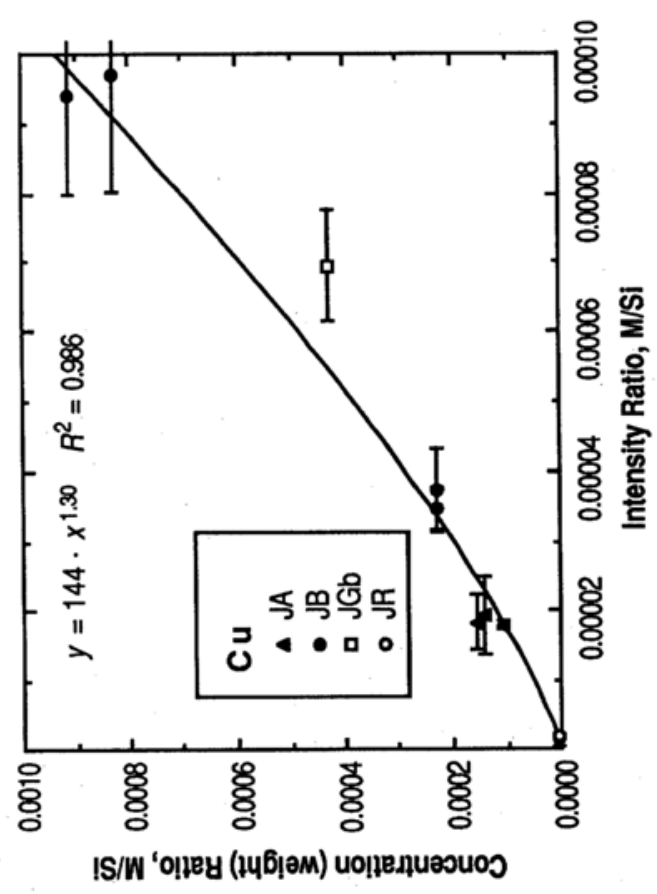

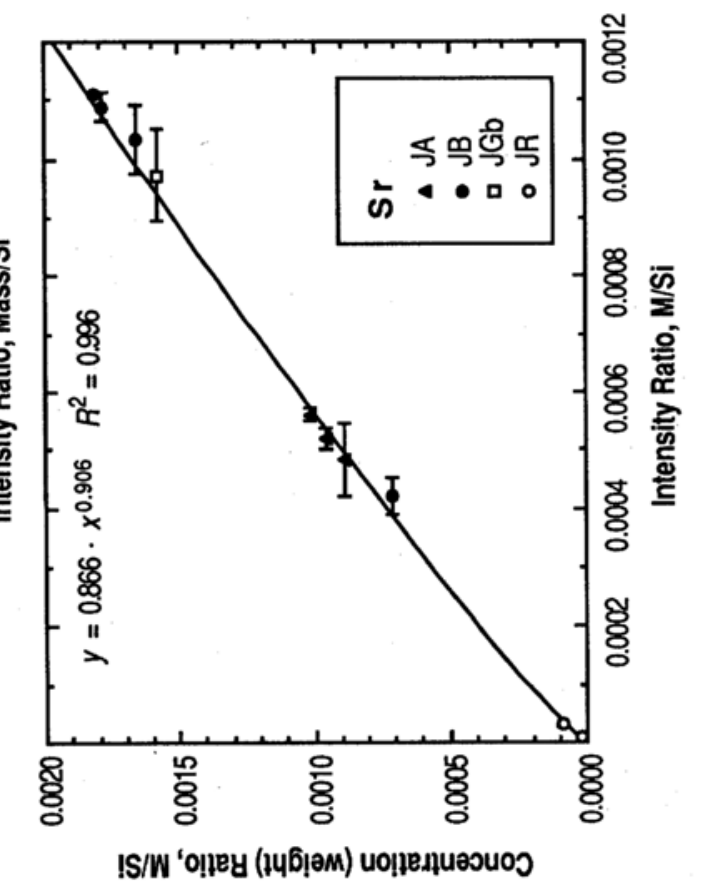

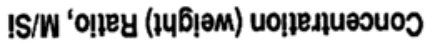

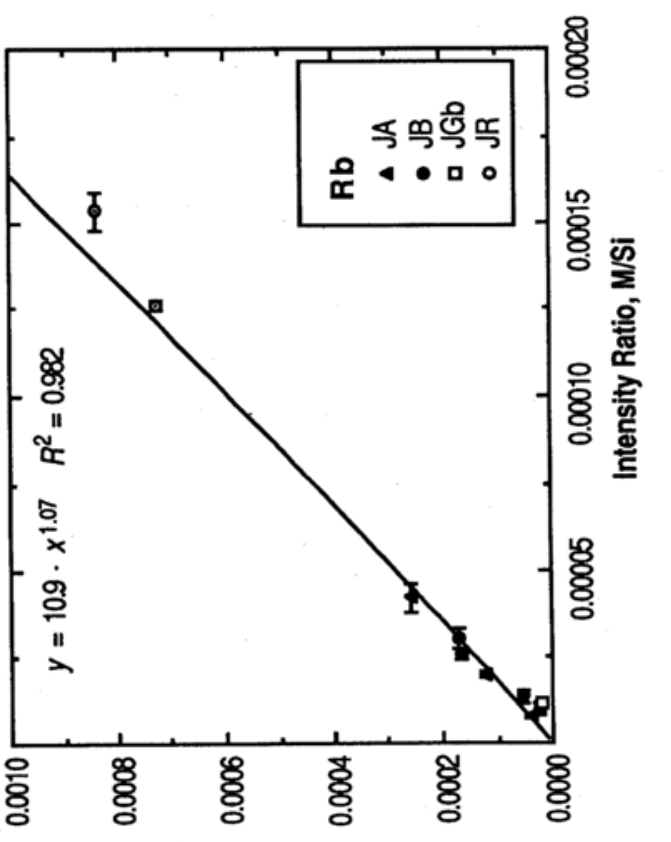

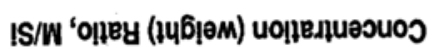



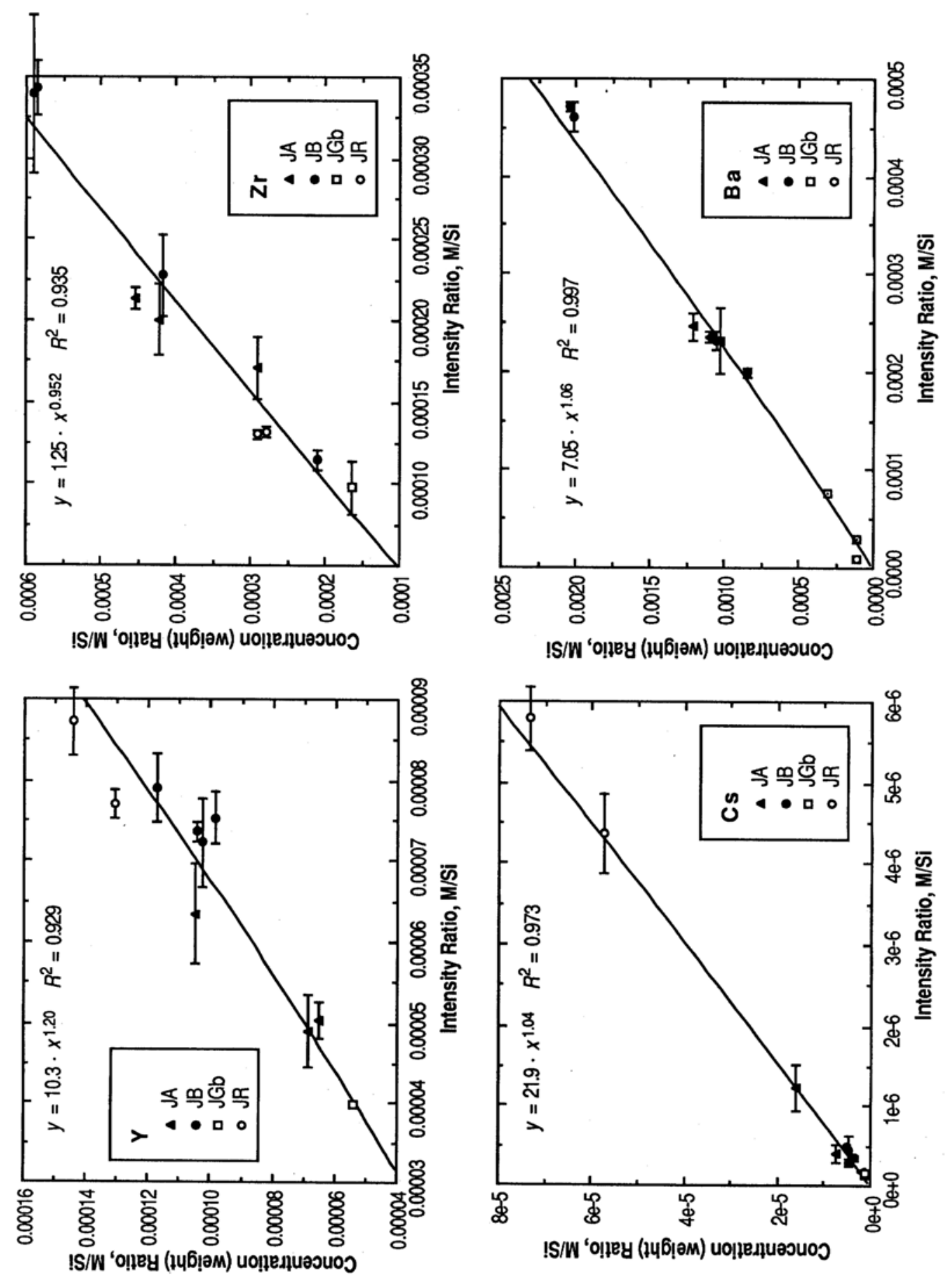


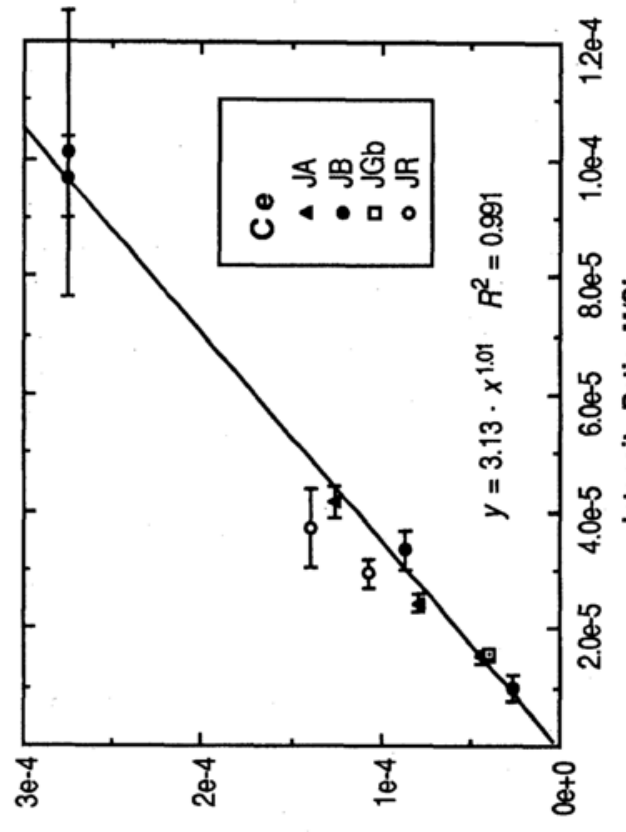

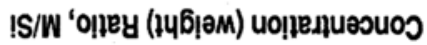

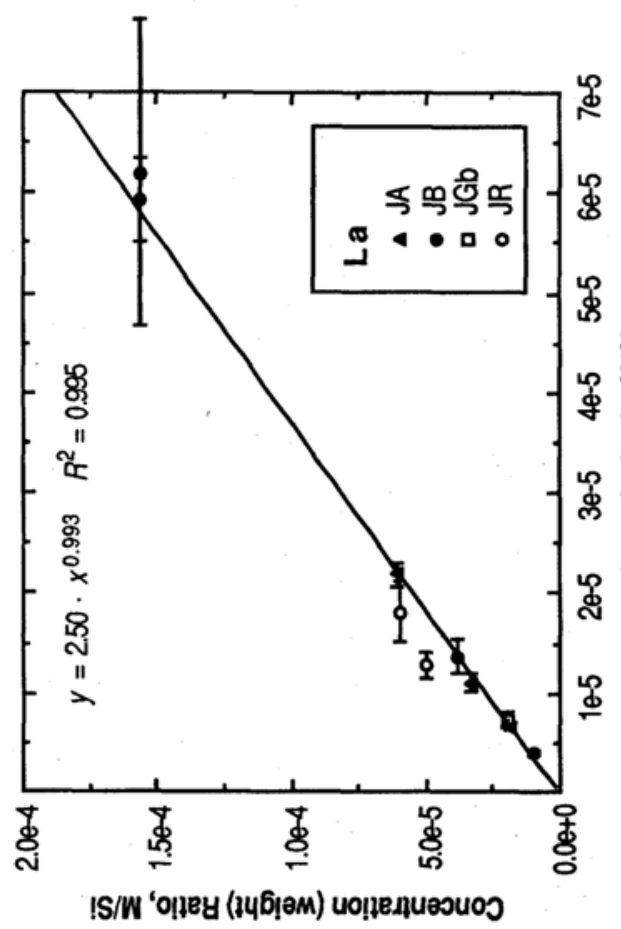

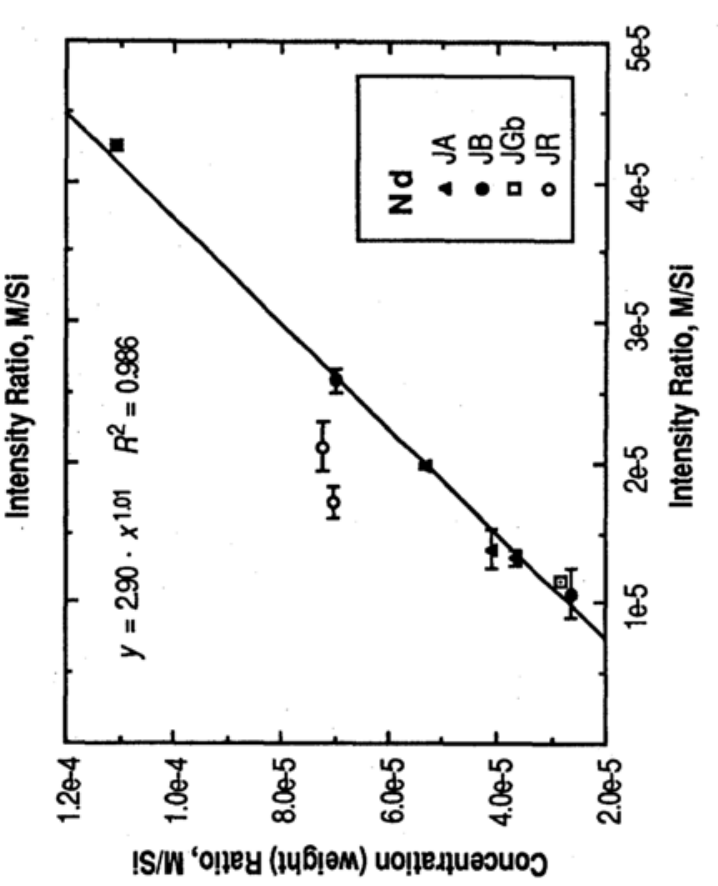

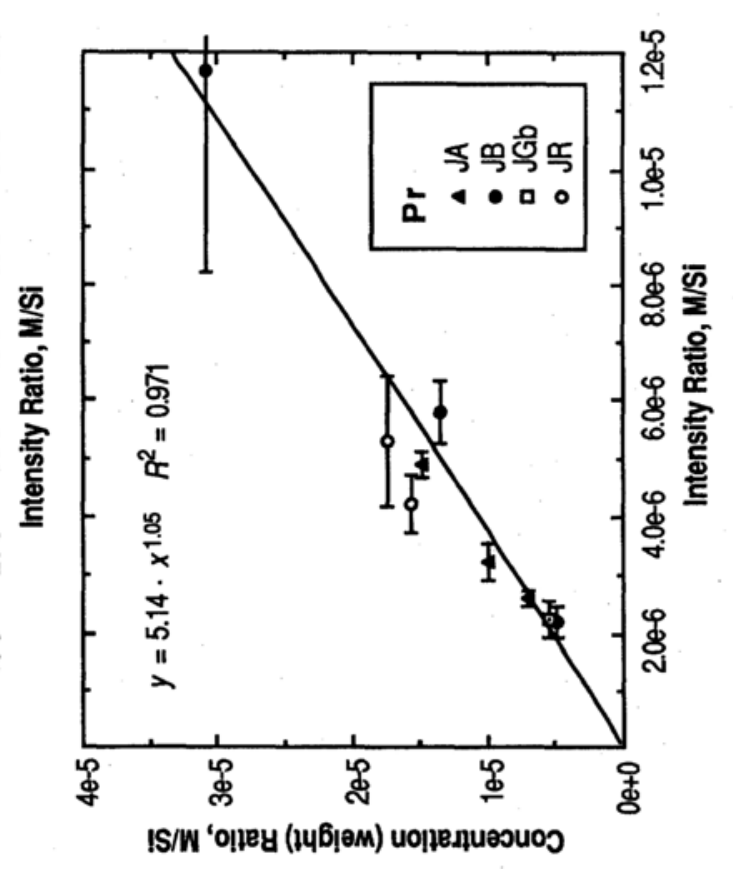




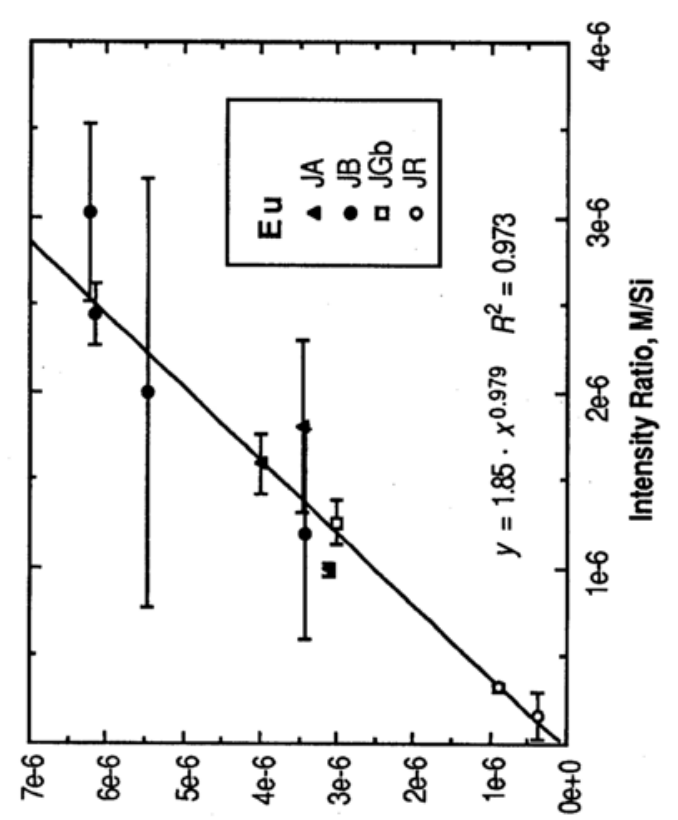

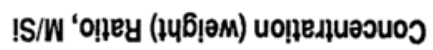

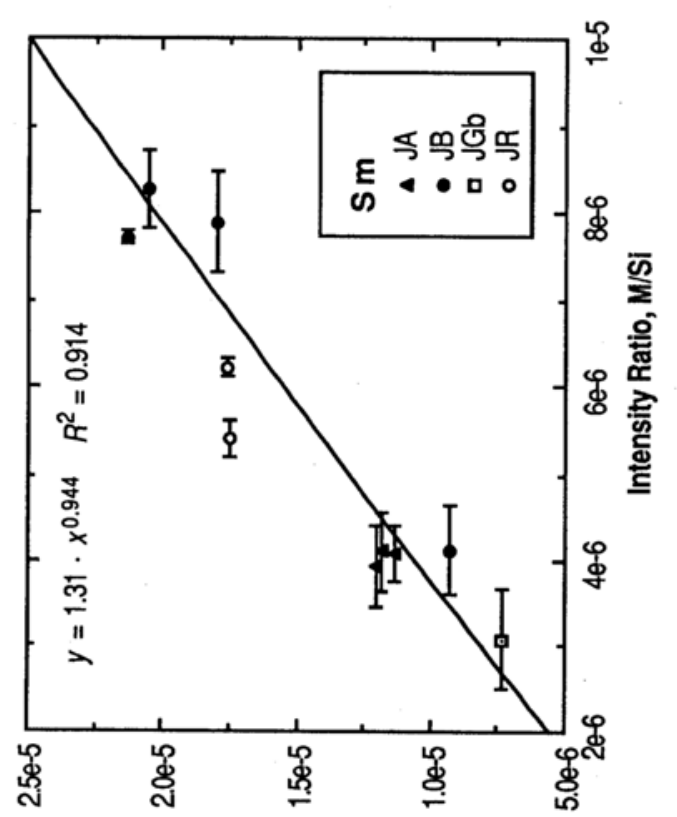

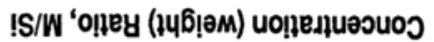

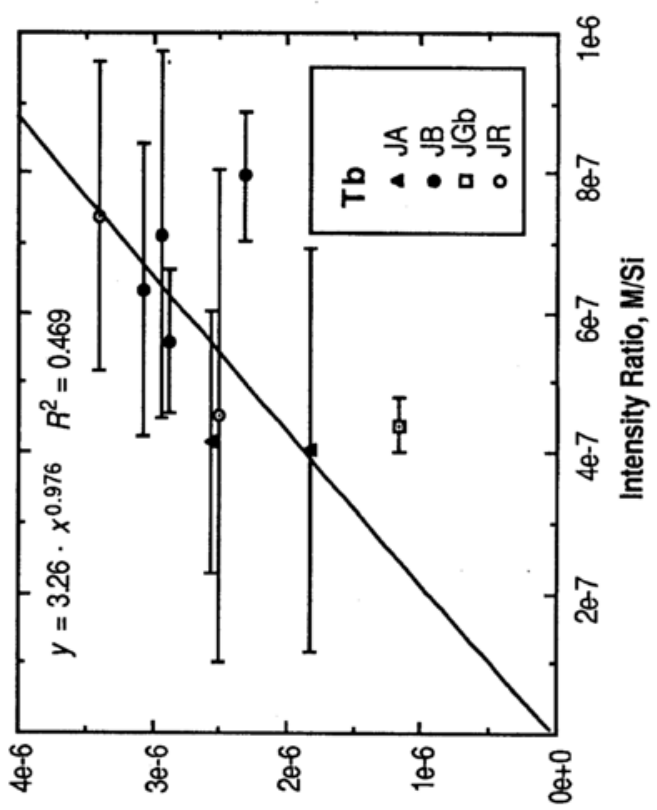

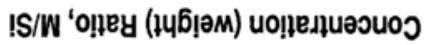

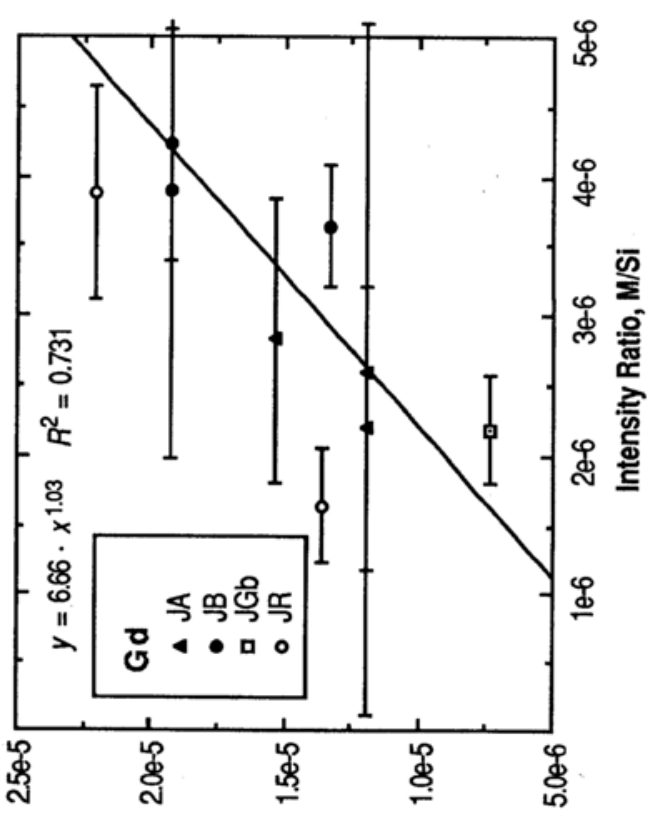

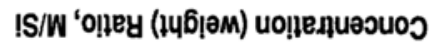




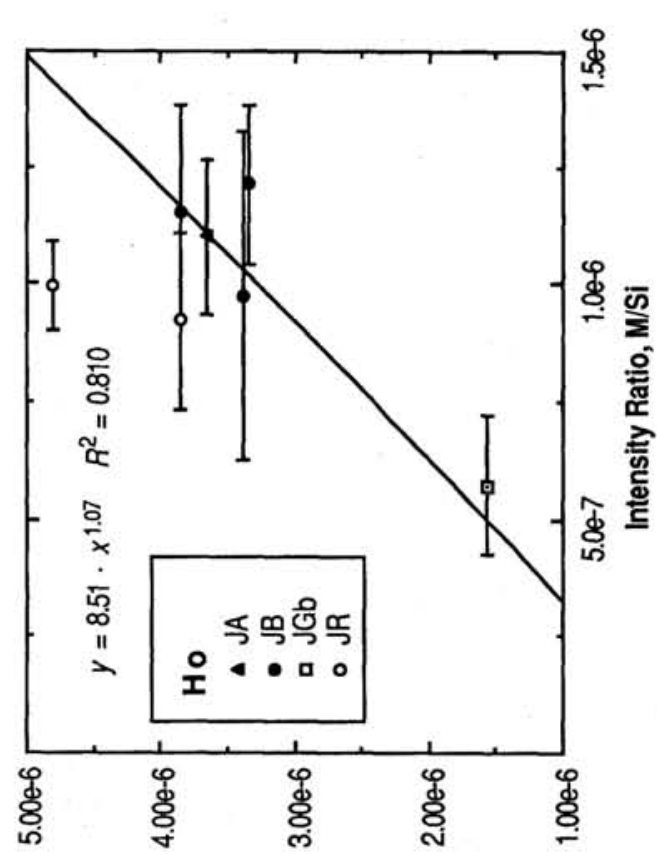

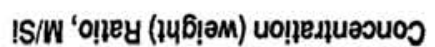

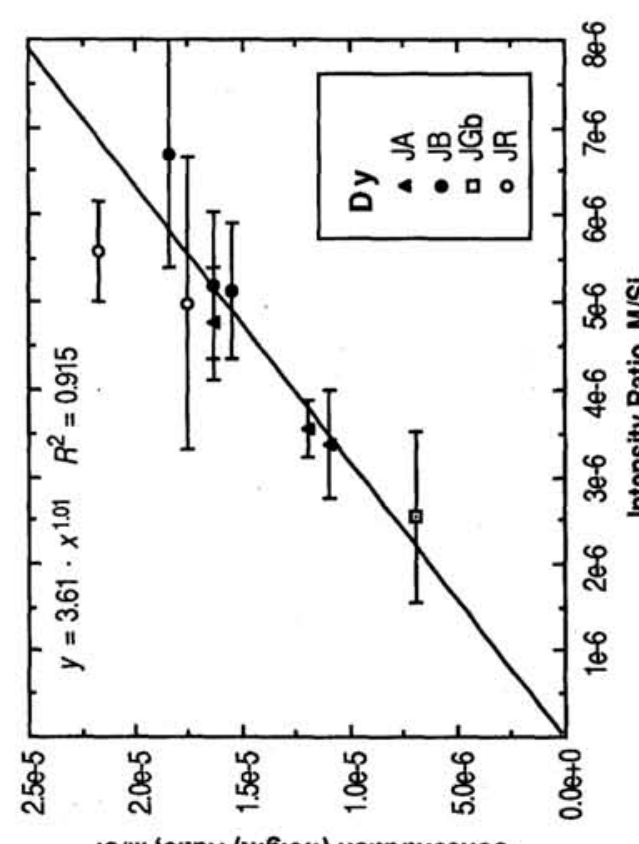

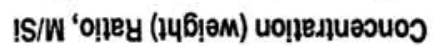
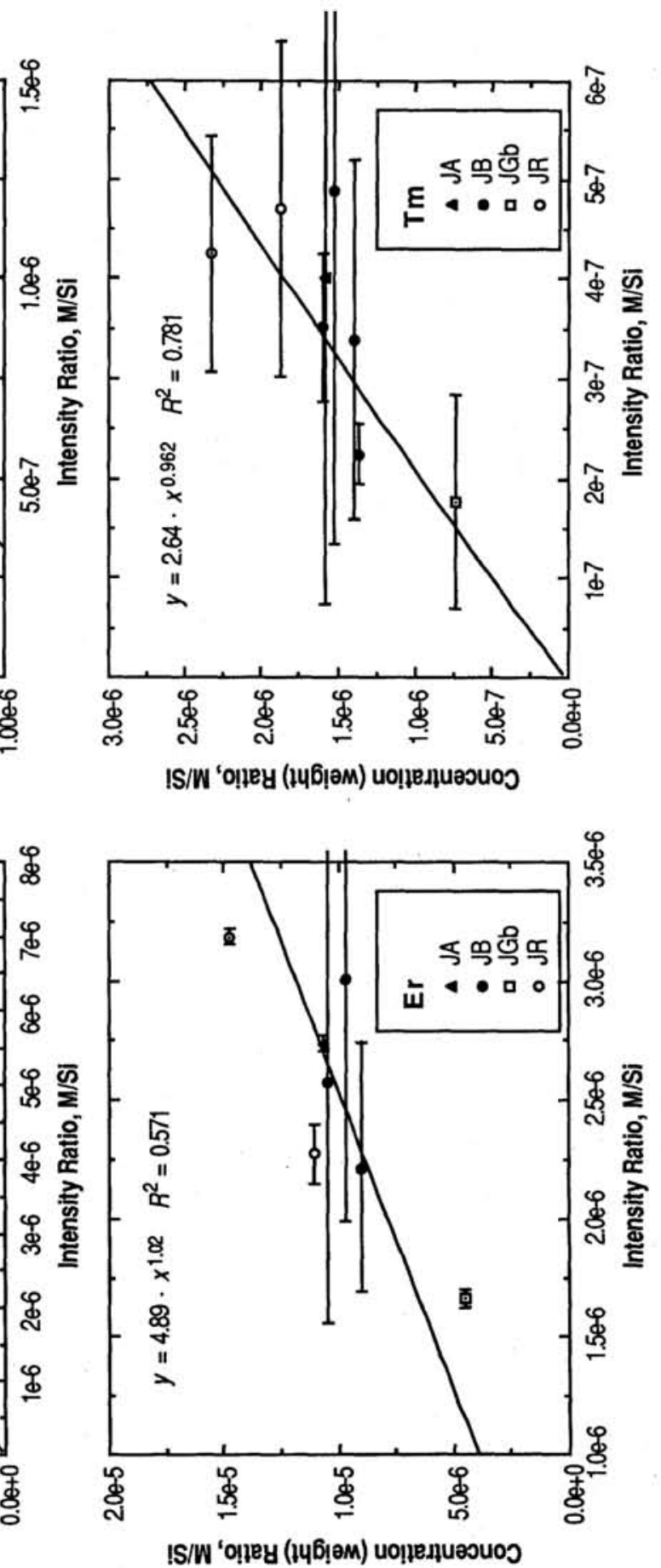
H. Yurimoto et al.
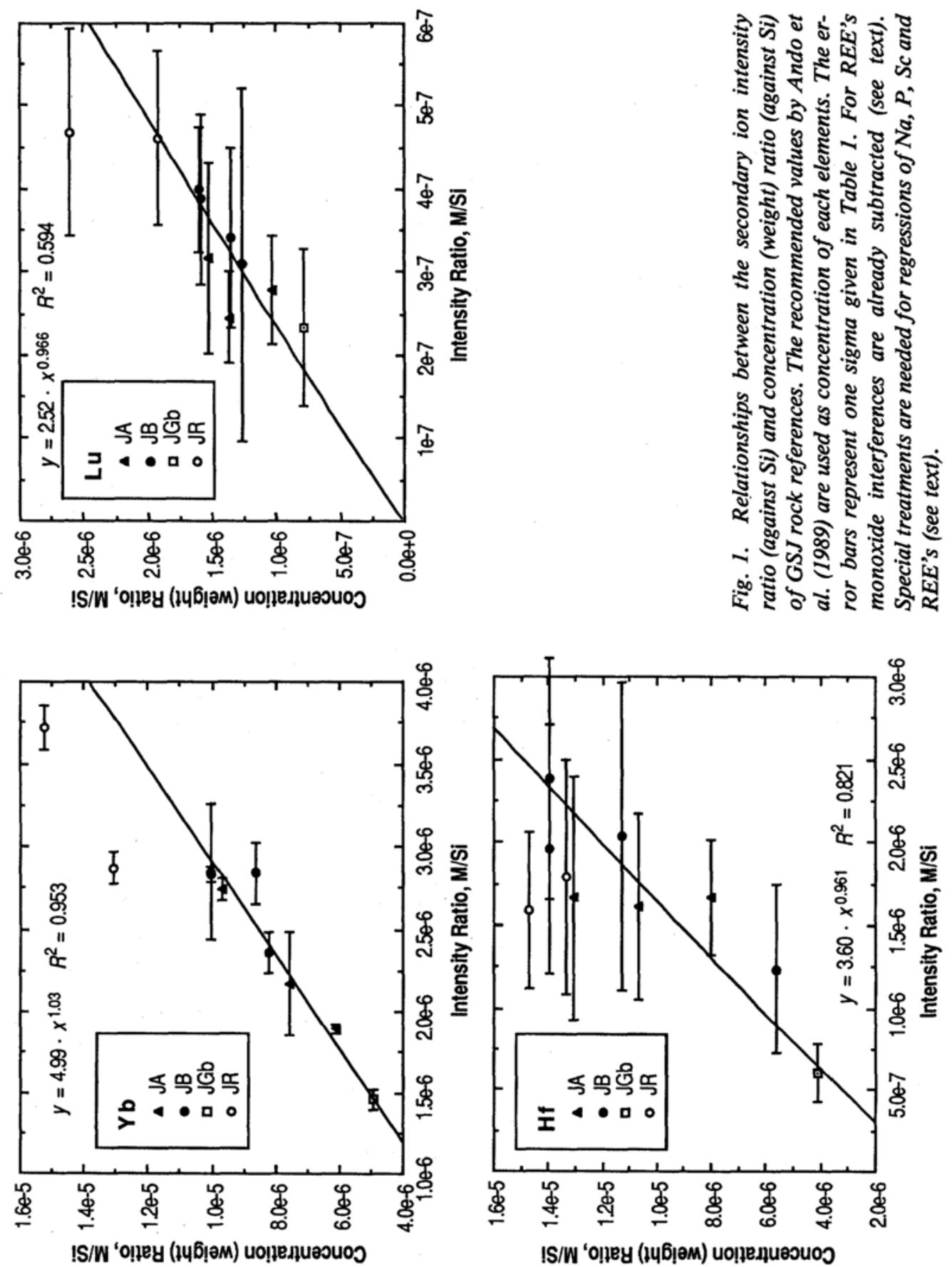
results of SIMS measurements for most major elements show no large discrepancies from the correlation curve of the homogeneous glass standards (Fig. 1), micro-scale compositional heterogeneity, greater than $50 \%$, was found for some trace elements (e.g. REE) in these glasses, and also in JF-2 and JG-3 glasses. As a consequence, these heterogeneous glasses were not used as standards for the empirical approach to quantitative SIMS.

\section{Quantification}

\section{General}

Isotopes used for quantification are listed in Table 2. Secondary ion intensities, corrected for natural isotopic abundances, were normalized to the intensity of Si. Relationships between the intensity ratio and concentration ratio (on the weight-for-weight basis) of recommended values (Ando et al., 1989) against $\mathrm{Si}$ are presented in Fig. 1.

The results were fitted by a simple linear regression and a logarithmic regression. The correlation coefficients for both regressions have similar values. However, the logarithmic regression minimizes the relative errors, and is therefore much more effective for elements whose concentration distributes over a range of several magnitudes. Only logarithmic regression lines are shown in Fig. 1.

All of the values of secondary ion intensity ratios produce linear relationships when plotted against concentration ratios within uncertainties of measurement. Moreover it seems that possible interferences are negligible over the concentration range of the glass standards for all elements except $\mathrm{P}, \mathrm{Sc}$, heavy rare earth elements (REE's) and Hf. Therefore, within the analytical uncertainties, the correlation of a given element $M$ can be simplified as:

$$
[M]=F_{M} \frac{M^{+}}{S i^{+}}[S i]
$$

where $[M]$ and [Si] are concentrations of $M$ and $\mathrm{Si}$, respectively, $\mathrm{M}^{+}$and $\mathrm{Si}^{+}$, secondary ion intensity ratios of individual element, and $F_{M}$, rela- tive sensitivity factor of $M$ to $\mathrm{Si}$. Since this simple relationship was well demonstrated for the individual element, the number of standards needed can be considerably reduced for practical purposes.

Comparisons of the SIMS measurements with the recommended values of Ando et al. (1989) are shown in Table 1. The agreement between SIMS and the recommended values are typically better than $10 \%$ accuracy level (see R.E. \% of Table 1).

\section{$\mathrm{Na}$}

Sodium ion intensities of Si-rich glass standards (JF- JG- and JR-series) have large discrepancies from a regression curve by the other glass standards (Fig. 1). The fact that the data points are on the right of the curve shows that there are more $\mathrm{Na}$ ions in these standards than expected (i.e., enhanced ionization). The matrix effects on $\mathrm{Na}$ ion intensities may be caused by the Si-induced enhancement (Shimizu, 1986).

Good correlations were obtained except for the Si-rich standards (Fig. 1). The relation is useful for quantification of the silica-poor materials (within 5\% systematic error), and for semi-quantification of the silica-rich material (within $30 \%$ systematic error) (Table 1).

\section{$P$ and $S c$}

Nonzero intercepts on Fig. 1 indicate that interferences still remained on the masses of $\mathrm{P}$ and Sc after energy filtering. Since these are monoisotopic elements, only the high mass resolution technique can produce an interference-free peak (Yurimoto and Sueno, 1984). According to the high mass resolution study, $\mathrm{SiO}^{+}, \mathrm{AlO}^{+}$and $\mathrm{CaH}^{+}$ions overlap with the $\mathrm{Sc}^{+}$peak, and $\mathrm{SiH}^{+}$ ion with the $\mathrm{P}^{+}$peak. The $\mathrm{CaH}^{+}$interference is negligible (less than $10 \%$ of $\mathrm{Sc}^{+}$peak under no energy filtering) in this study.

Since these interferences seem to be contributing constantly to all standards under this SIMS condition, simple linear regressions were applied to these elements (Fig. 1). We obtained good correlation coefficients $R^{2}=0.974$ and 
Table 1. Results of SIMS analysis of the glass standards

\begin{tabular}{|c|c|c|c|c|c|c|c|c|c|c|}
\hline & \multicolumn{5}{|c|}{ JA-1 } & \multicolumn{5}{|c|}{ JA-2 } \\
\hline & \multirow{2}{*}{$\frac{\text { GSJ88 }}{\text { ppmw }}$} & \multicolumn{4}{|c|}{ SIMS } & \multirow{2}{*}{$\frac{\text { GSJ88 }}{\text { ppmw }}$} & \multicolumn{4}{|c|}{ SIMS } \\
\hline & & ppmw & sigma & CV\% & R.E.\% & & ppmw & sigma & $\mathrm{CV} \%$ & R.E. \% \\
\hline $\mathrm{Li}$ & 10.5 & 11.7 & 0.8 & $7 \%$ & $-11 \%$ & 28.7 & 23.1 & 0.3 & $1 \%$ & $19 \%$ \\
\hline $\mathrm{Be}$ & 0.5 & 0.49 & 0.03 & $6 \%$ & $1 \%$ & 2.2 & 2.1 & 0.3 & $14 \%$ & $4 \%$ \\
\hline B & 16 & 23 & 1 & $3 \%$ & $-44 \%$ & & 17 & 0 & $2 \%$ & $\cdot$ \\
\hline $\mathrm{Na}$ & 28606 & 30563 & 1242 & $4 \%$ & $-7 \%$ & 22825 & 21251 & 916 & $4 \%$ & $7 \%$ \\
\hline $\mathrm{Mg}$ & 9705 & 10687 & 1743 & $16 \%$ & $-10 \%$ & 46295 & 46141 & 8026 & $17 \%$ & $0 \%$ \\
\hline $\mathrm{Al}$ & 79298 & 82417 & 2307 & $3 \%$ & $-4 \%$ & 81098 & 79459 & 2359 & $3 \%$ & $2 \%$ \\
\hline $\mathrm{Si}$ & 299560 & 299560 & & & & 262711 & 262711 & & & \\
\hline $\mathbf{P}$ & 698 & 741 & 25 & $3 \%$ & $-6 \%$ & 654 & 545 & 4 & $1 \%$ & $17 \%$ \\
\hline $\mathbf{K}$ & 6475 & 6486 & 451 & $7 \%$ & $0 \%$ & 14942 & 12755 & 1450 & $11 \%$ & $15 \%$ \\
\hline $\mathrm{Ca}$ & 40593 & 40251 & 3080 & $8 \%$ & $1 \%$ & 46310 & 45382 & 2676 & $6 \%$ & $2 \%$ \\
\hline Sc & 28.4 & 21.9 & 3.3 & $15 \%$ & $23 \%$ & 19 & 19.1 & 0.4 & $2 \%$ & $-1 \%$ \\
\hline $\mathrm{Ti}$ & 5213 & 5175 & 524 & $10 \%$ & $1 \%$ & 4015 & 4172 & 182 & $4 \%$ & $-4 \%$ \\
\hline V & 105 & 91 & 8 & $9 \%$ & $14 \%$ & 130 & 146 & 3 & $2 \%$ & $-12 \%$ \\
\hline $\mathrm{Cr}$ & 7.3 & 5.6 & 2.3 & $40 \%$ & $23 \%$ & 465 & 388 & 5 & $1 \%$ & $17 \%$ \\
\hline Mn & 1162 & 1078 & 276 & $26 \%$ & $7 \%$ & 852 & 895 & 103 & $12 \%$ & $-5 \%$ \\
\hline $\mathrm{Fe}$ & 48645 & 48551 & 4172 & $9 \%$ & $0 \%$ & 42885 & 45392 & 7597 & $17 \%$ & $-6 \%$ \\
\hline Co & 11.8 & 11.4 & 2.6 & $23 \%$ & $4 \%$ & 30 & 29 & 2 & $6 \%$ & $3 \%$ \\
\hline $\mathrm{Cu}$ & 42.2 & 33.0 & 12.5 & $38 \%$ & $22 \%$ & 28.6 & 26.5 & 1.0 & $4 \%$ & $7 \%$ \\
\hline $\mathbf{R b}$ & 11.8 & 12.3 & 1.5 & $12 \%$ & $-4 \%$ & 68 & 61 & 7 & $11 \%$ & $10 \%$ \\
\hline $\mathrm{Sr}$ & 266 & 258 & 31 & $12 \%$ & $3 \%$ & 252 & 242 & 7 & $3 \%$ & $4 \%$ \\
\hline $\mathbf{Y}$ & 31.4 & 27.6 & 3.2 & $12 \%$ & $12 \%$ & 18 & 18 & 2 & $11 \%$ & $1 \%$ \\
\hline $\mathrm{Zr}$ & 87 & 97 & 10 & $10 \%$ & $-12 \%$ & 119 & 105 & 3 & $3 \%$ & $12 \%$ \\
\hline Cs & 0.64 & 0.62 & 0.00 & $1 \%$ & $3 \%$ & 4.2 & 4.1 & 1.0 & $25 \%$ & $3 \%$ \\
\hline $\mathrm{Ba}$ & 307 & 306 & 47 & $15 \%$ & $0 \%$ & 317 & 285 & 17 & $6 \%$ & $10 \%$ \\
\hline $\mathrm{La}$ & 5.5 & 5.3 & 0.2 & $4 \%$ & $5 \%$ & 16 & 15.5 & 0.8 & $5 \%$ & $3 \%$ \\
\hline $\mathrm{Ce}$ & 13.2 & 12.6 & 1.0 & $8 \%$ & $5 \%$ & 33 & 30.8 & 2.0 & $6 \%$ & $7 \%$ \\
\hline Pr & 1.5 & 2.0 & 0.1 & $6 \%$ & $-\mathbf{3 3} \%$ & 5.9 & 3.4 & 0.2 & $5 \%$ & $42 \%$ \\
\hline $\mathrm{Nd}$ & 11 & 10.4 & 0.4 & $4 \%$ & $5 \%$ & 14 & 13.8 & 0.1 & $1 \%$ & $2 \%$ \\
\hline $\mathrm{Sm}$ & 3.6 & 3.1 & 0.3 & $11 \%$ & $14 \%$ & 3.1 & 2.8 & 0.3 & $11 \%$ & $9 \%$ \\
\hline $\mathrm{Eu}$ & 1.2 & 1.2 & 0.1 & $10 \%$ & $2 \%$ & 0.91 & 1.16 & 0.31 & $27 \%$ & $-28 \%$ \\
\hline Gd & 4.6 & 3.8 & 1.4 & $37 \%$ & $17 \%$ & 3.9 & 3.1 & 3.0 & $99 \%$ & $21 \%$ \\
\hline $\mathrm{Tb}$ & 0.77 & 0.57 & 0.25 & $44 \%$ & $26 \%$ & 0.48 & 0.49 & 0.34 & $69 \%$ & $-2 \%$ \\
\hline Dy & 4.9 & 4.5 & 0.6 & $14 \%$ & $9 \%$ & 1 & 2.9 & 0.3 & $9 \%$ & $-192 \%$ \\
\hline Ho & 0.89 & 1.08 & 0.17 & $16 \%$ & $-21 \%$ & & 0.64 & 0.10 & $15 \%$ & \\
\hline $\mathrm{Er}$ & 3.2 & 3.2 & 0.0 & $1 \%$ & $0 \%$ & & 1.6 & 0.4 & $26 \%$ & \\
\hline $\mathrm{Tm}$ & 0.51 & 0.55 & 0.43 & $79 \%$ & $-8 \%$ & & 0.32 & 0.13 & $42 \%$ & \\
\hline $\mathrm{Yb}$ & 2.9 & 2.8 & 0.1 & $2 \%$ & $3 \%$ & 1.6 & 1.7 & 0.0 & $2 \%$ & $-5 \%$ \\
\hline $\mathrm{Lu}$ & 0.46 & 0.40 & 0.14 & $35 \%$ & $14 \%$ & 0.27 & 0.31 & 0.07 & $23 \%$ & $-13 \%$ \\
\hline Hf & 2.4 & 3.0 & 0.6 & $20 \%$ & $-25 \%$ & 2.8 & 2.6 & 0.9 & $33 \%$ & $9 \%$ \\
\hline
\end{tabular}


Table 1. (continued)

\begin{tabular}{|c|c|c|c|c|c|c|c|c|c|c|}
\hline & \multicolumn{5}{|c|}{ JA-3 } & \multicolumn{5}{|c|}{ JB-1 } \\
\hline & \multirow{2}{*}{$\frac{\text { GSJ88 }}{\text { ppmw }}$} & \multicolumn{4}{|c|}{ SIMS } & \multirow{2}{*}{$\frac{\text { GSJ88 }}{\text { ppmw }}$} & \multicolumn{4}{|c|}{ SIMS } \\
\hline & & ppmw & sigma & $\mathrm{CV} \%$ & R.E.\% & & ppmw & sigma & $\mathrm{CV} \%$ & R.E.\% \\
\hline $\mathrm{Li}$ & 14 & 13.2 & 0.0 & $0 \%$ & $6 \%$ & 11.5 & 10.3 & 0.4 & $4 \%$ & $10 \%$ \\
\hline $\mathrm{Be}$ & 0.7 & 0.77 & 0.12 & $16 \%$ & $-10 \%$ & 1.5 & 1.37 & 0.04 & $3 \%$ & $9 \%$ \\
\hline B & & 23 & 1 & $2 \%$ & & 12.4 & 9.1 & 0.6 & $7 \%$ & $27 \%$ \\
\hline $\mathrm{Na}$ & 23492 & 23367 & 2392 & $10 \%$ & $1 \%$ & 20676 & 20558 & 1223 & $6 \%$ & $1 \%$ \\
\hline $\mathrm{Mg}$ & 22002 & 24203 & 9448 & $39 \%$ & $-10 \%$ & 46597 & 42313 & 4394 & $10 \%$ & $9 \%$ \\
\hline Al & 82422 & 80250 & 5020 & $6 \%$ & $3 \%$ & 76916 & 78346 & 3189 & $4 \%$ & $-2 \%$ \\
\hline$S i$ & 291143 & 291143 & & & & 243959 & 243959 & & & \\
\hline $\mathbf{P}$ & 480 & 532 & 70 & $13 \%$ & $-11 \%$ & 1134 & 1109 & 66 & $6 \%$ & $2 \%$ \\
\hline $\mathbf{K}$ & 11705 & 10967 & 1634 & $15 \%$ & $6 \%$ & 11871 & 11407 & 806 & $7 \%$ & $4 \%$ \\
\hline $\mathrm{Ca}$ & 44880 & 43663 & 4496 & $10 \%$ & $3 \%$ & 66392 & 63891 & 4184 & $7 \%$ & $4 \%$ \\
\hline Sc & 21 & 26 & 4 & $14 \%$ & $-\mathbf{2 5} \%$ & 27.4 & 28.9 & 1.6 & $5 \%$ & $-6 \%$ \\
\hline $\mathrm{Ti}$ & 4075 & 3866 & 383 & $10 \%$ & $5 \%$ & 8030 & 7403 & 596 & $8 \%$ & $8 \%$ \\
\hline V & 172 & 177 & 1 & $1 \%$ & $-3 \%$ & 212 & 251 & 16 & $6 \%$ & $-18 \%$ \\
\hline $\mathrm{Cr}$ & 67.5 & 78 & 13 & $17 \%$ & $-16 \%$ & 469 & 419 & 26 & $6 \%$ & $11 \%$ \\
\hline Mn & 821 & 871 & 203 & $23 \%$ & $-6 \%$ & 1239 & 1004 & 228 & $23 \%$ & $19 \%$ \\
\hline $\mathrm{Fe}$ & 46089 & 45392 & 13458 & $30 \%$ & $2 \%$ & 62591 & 55263 & 8735 & $16 \%$ & $12 \%$ \\
\hline $\mathrm{Co}$ & 21 & 26 & 5 & $21 \%$ & $-26 \%$ & 38.7 & 36.4 & 0.8 & $2 \%$ & $6 \%$ \\
\hline $\mathrm{Cu}$ & 45.3 & 30.2 & 8.6 & $29 \%$ & $33 \%$ & 56.3 & 63.2 & 12.8 & $20 \%$ & $-12 \%$ \\
\hline $\mathbf{R b}$ & 36 & 31 & 1 & $3 \%$ & $14 \%$ & 41.2 & 40.8 & 4.2 & $10 \%$ & $1 \%$ \\
\hline $\mathrm{Sr}$ & 294 & 287 & 5 & $2 \%$ & $2 \%$ & 435 & 439 & 9 & $2 \%$ & $-1 \%$ \\
\hline $\mathbf{Y}$ & 19 & 20 & 1 & $5 \%$ & $-7 \%$ & 24 & 28 & 1 & $5 \%$ & $-15 \%$ \\
\hline $\mathrm{Zr}$ & 123 & 110 & 11 & $10 \%$ & $11 \%$ & 143 & 154 & 7 & $5 \%$ & $-7 \%$ \\
\hline Cs & 2.2 & 1.4 & 0.4 & $29 \%$ & $36 \%$ & 1.19 & 1.29 & 0.59 & $46 \%$ & $-9 \%$ \\
\hline $\mathrm{Ba}$ & 318 & 303 & 7 & $2 \%$ & $5 \%$ & 490 & 516 & 18 & $3 \%$ & $-5 \%$ \\
\hline $\mathrm{La}$ & 9.7 & 8.7 & 0.6 & $7 \%$ & $10 \%$ & 38 & 40.4 & 9.8 & $24 \%$ & $-6 \%$ \\
\hline $\mathrm{Ce}$ & 23 & 19.8 & 1.2 & $6 \%$ & $14 \%$ & 67 & 70.0 & 17.1 & $24 \%$ & $-5 \%$ \\
\hline $\operatorname{Pr}$ & 2.9 & 2.4 & 0.2 & $10 \%$ & $16 \%$ & 7.5 & 7.9 & 2.5 & $31 \%$ & $-5 \%$ \\
\hline $\mathrm{Nd}$ & 12 & 10.7 & 1.1 & $10 \%$ & $11 \%$ & 27 & 27.7 & 0.2 & $1 \%$ & $-2 \%$ \\
\hline $\mathrm{Sm}$ & 3.3 & 3.1 & 0.2 & $8 \%$ & $6 \%$ & 5 & 5.1 & 0.3 & $5 \%$ & $-2 \%$ \\
\hline $\mathrm{Eu}$ & 0.9 & 0.72 & 0.03 & $4 \%$ & $20 \%$ & 1.52 & 1.79 & 0.30 & $17 \%$ & $-18 \%$ \\
\hline Gd & 2 & 2.9 & 1.3 & $47 \%$ & $-43 \%$ & 4.7 & 4.7 & 1.0 & $20 \%$ & $0 \%$ \\
\hline $\mathrm{Tb}$ & & 0.80 & 0.39 & $49 \%$ & & 0.76 & 0.79 & 0.28 & $36 \%$ & $-4 \%$ \\
\hline Dy & 3.2 & 3.1 & 0.6 & $19 \%$ & $4 \%$ & 4 & 4.0 & 0.6 & $16 \%$ & $0 \%$ \\
\hline Ho & & 0.66 & 0.28 & $42 \%$ & & 0.7 & 0.77 & 0.30 & $38 \%$ & $-10 \%$ \\
\hline $\mathrm{Er}$ & & 1.7 & 0.3 & $20 \%$ & & 2.2 & 2.1 & 0.5 & $24 \%$ & $4 \%$ \\
\hline $\mathrm{Tm}$ & & 0.53 & 0.33 & $62 \%$ & & 0.35 & 0.26 & 0.03 & $13 \%$ & $27 \%$ \\
\hline $\mathrm{Yb}$ & 2.2 & 2.1 & 0.3 & $15 \%$ & $3 \%$ & 2.1 & 2.4 & 0.2 & $7 \%$ & $-13 \%$ \\
\hline Lu & 0.4 & 0.30 & 0.06 & $22 \%$ & $25 \%$ & 0.31 & 0.31 & 0.21 & $66 \%$ & $-1 \%$ \\
\hline Hf & 3.8 & 2.9 & 1.2 & $42 \%$ & $23 \%$ & 3.4 & 2.9 & 1.1 & $37 \%$ & $16 \%$ \\
\hline
\end{tabular}


H. Yurimoto et al.

Table 1. (continued)

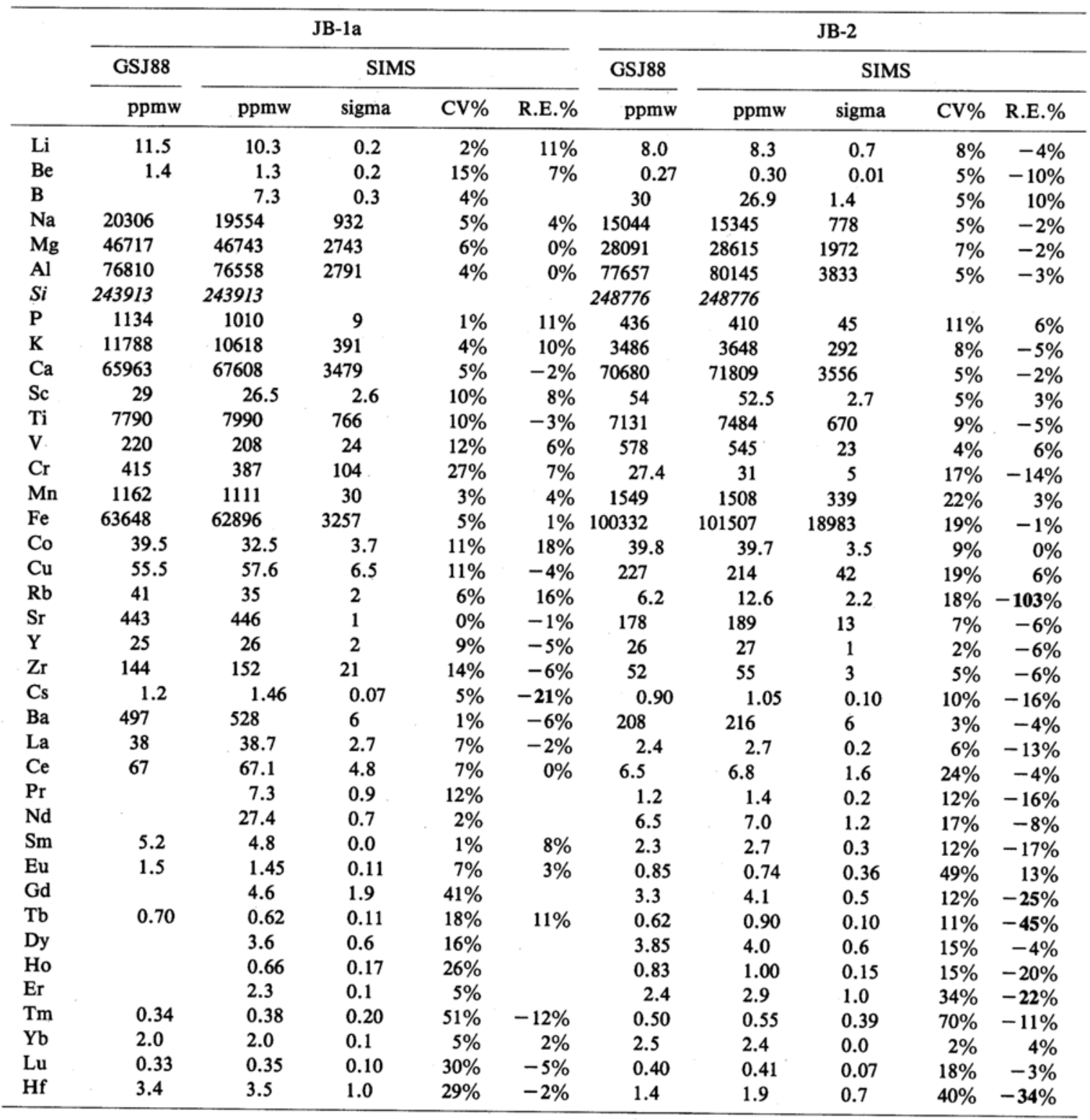


Table 1. (continued)

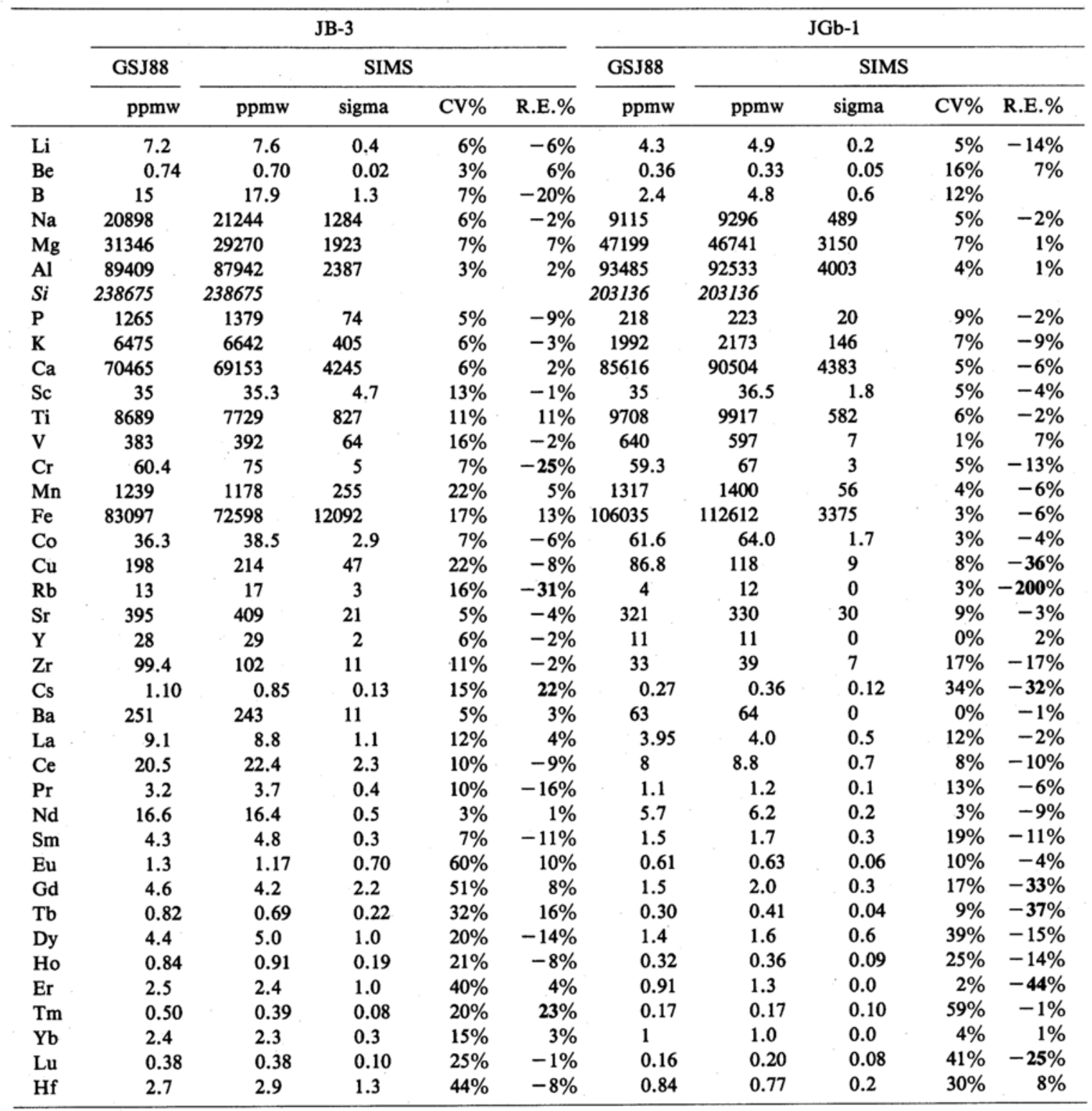


Table 1. (continued)

\begin{tabular}{|c|c|c|c|c|c|c|c|c|c|c|}
\hline & \multicolumn{5}{|c|}{ JR-1 } & \multicolumn{5}{|c|}{ JR-2 } \\
\hline & \multirow{2}{*}{$\frac{\text { GSJ88 }}{\text { ppmw }}$} & \multicolumn{4}{|c|}{ SIMS } & \multirow{2}{*}{$\frac{\text { GSJ88 }}{\text { ppmw }}$} & \multicolumn{4}{|c|}{ SIMS } \\
\hline & & ppmw & sigma & CV\% & R.E.\% & & ppmw & sigma & CV\% & R.E.\% \\
\hline $\mathrm{Li}$ & 62.3 & 69 & 5 & $8 \%$ & $-11 \%$ & 83 & 88 & 10 & $11 \%$ & $-6 \%$ \\
\hline $\mathrm{Be}$ & 3.1 & 3.4 & 0.3 & $7 \%$ & $-9 \%$ & 3.4 & 3.6 & 0.4 & $10 \%$ & $-7 \%$ \\
\hline B & 133 & 115 & 2 & $1 \%$ & $14 \%$ & 135 & 137 & 1 & $1 \%$ & $-2 \%$ \\
\hline $\mathrm{Na}$ & 30384 & 38133 & 2987 & $8 \%$ & $-26 \%$ & 29866 & 37861 & 2429 & $6 \%$ & $-27 \%$ \\
\hline $\mathrm{Mg}$ & 543 & 558 & 241 & $43 \%$ & $-3 \%$ & 301 & 282 & 60 & $21 \%$ & $6 \%$ \\
\hline $\mathrm{Al}$ & 68234.7 & 68041 & 3391 & $5 \%$ & $0 \%$ & 67864 & 67213 & 2801 & $4 \%$ & $1 \%$ \\
\hline$S i$ & 352635 & 352635 & & & & 353758 & 353758 & & & \\
\hline $\mathbf{P}$ & 87 & 175 & 36 & $20 \%$ & $-101 \%$ & 44 & 63 & 37 & $59 \%$ & $-43 \%$ \\
\hline $\mathbf{K}$ & 36608 & 40662 & 1517 & $4 \%$ & $-11 \%$ & 36940 & 40741 & 1653 & $4 \%$ & $-10 \%$ \\
\hline $\mathrm{Ca}$ & 4502 & 4177 & 543 & $13 \%$ & $7 \%$ & 3216 & 3538 & 348 & $10 \%$ & $-10 \%$ \\
\hline $\mathrm{Sc}$ & 5.2 & 5.7 & 0.7 & $13 \%$ & $-10 \%$ & 5.4 & 6.5 & 0.7 & $11 \%$ & $-20 \%$ \\
\hline $\mathrm{Ti}$ & 599 & 779 & 445 & $57 \%$ & $-\mathbf{3 0} \%$ & 539 & 431 & 27 & $6 \%$ & $20 \%$ \\
\hline V & & 2.2 & 0.4 & $19 \%$ & $\mathbf{7 3} \%$ & & 1.3 & 0.1 & $7 \%$ & $84 \%$ \\
\hline $\mathrm{Cr}$ & 2.3 & 0.28 & 0.08 & $27 \%$ & $\mathbf{8 8} \%$ & 2.6 & 0.09 & 0.02 & $20 \%$ & $\mathbf{9 7} \%$ \\
\hline Mn & 775 & 755 & 182 & $24 \%$ & $3 \%$ & 852 & 851 & 63 & $7 \%$ & $0 \%$ \\
\hline $\mathrm{Fe}$ & 6685 & 7772 & 4273 & $55 \%$ & $-16 \%$ & 6001 & 5927 & 444 & $7 \%$ & $1 \%$ \\
\hline Co & 0.65 & 2.1 & 0.7 & $33 \%$ & $-225 \%$ & 0.4 & 1.7 & 0.2 & $10 \%$ & $-323 \%$ \\
\hline $\mathrm{Cu}$ & 1.4 & 2.0 & 0.4 & $20 \%$ & $-40 \%$ & 1.4 & 1.3 & 1.1 & $84 \%$ & $9 \%$ \\
\hline $\mathrm{Rb}$ & 257 & 265 & 3 & $1 \%$ & $-3 \%$ & 297 & 328 & 12 & $4 \%$ & $-11 \%$ \\
\hline $\mathrm{Sr}$ & 30 & 25 & 2 & $7 \%$ & $18 \%$ & 8 & 9.4 & 0.1 & $1 \%$ & $-17 \%$ \\
\hline $\mathrm{Y}$ & 46 & 41 & 1 & $3 \%$ & $11 \%$ & 51 & 48 & 3 & $6 \%$ & $6 \%$ \\
\hline $\mathrm{Zr}$ & 102 & 88 & 2 & $2 \%$ & $13 \%$ & 98.5 & 89.7 & 2.3 & $3 \%$ & $9 \%$ \\
\hline Cs & 20.2 & 20.4 & 2.4 & $12 \%$ & $-1 \%$ & 26 & 27.5 & 1.9 & $7 \%$ & $-6 \%$ \\
\hline $\mathrm{Ba}$ & 40 & 41.1 & 0.2 & $1 \%$ & $-3 \%$ & 39 & 11.5 & 1.9 & $17 \%$ & $\mathbf{7 1} \%$ \\
\hline $\mathrm{La}$ & 21 & 17.2 & 2.9 & $17 \%$ & $18 \%$ & 17.5 & 12.2 & 1.3 & $11 \%$ & $\mathbf{3 0} \%$ \\
\hline $\mathrm{Ce}$ & 49 & 36.8 & 6.7 & $18 \%$ & $\mathbf{2 5} \%$ & 38 & 29.1 & 2.6 & $9 \%$ & $23 \%$ \\
\hline $\operatorname{Pr}$ & 6.1 & 5.0 & 1.1 & $22 \%$ & $19 \%$ & 5.5 & 3.9 & 0.5 & $12 \%$ & $\mathbf{2 9} \%$ \\
\hline Nd & 25.5 & 19.8 & 1.7 & $9 \%$ & $22 \%$ & 24.8 & 16.1 & 1.1 & $7 \%$ & $\mathbf{3 5} \%$ \\
\hline $\mathrm{Sm}$ & 6.2 & 5.6 & 0.1 & $2 \%$ & $\mathbf{9 \%}$ & 6.2 & 4.9 & 0.2 & $4 \%$ & $20 \%$ \\
\hline $\mathrm{Eu}$ & 0.31 & 0.29 & 0.02 & $6 \%$ & $7 \%$ & 0.13 & 0.15 & 0.12 & $82 \%$ & $-17 \%$ \\
\hline Gd & 4.8 & 2.6 & 0.7 & $26 \%$ & $\mathbf{4 6} \%$ & 7.8 & 6.3 & 1.3 & $20 \%$ & $20 \%$ \\
\hline $\mathrm{Tb}$ & 1.10 & 0.74 & 0.56 & $76 \%$ & $\mathbf{3 3} \%$ & 1.20 & 1.19 & 0.35 & $29 \%$ & $1 \%$ \\
\hline Dy & 6.2 & 5.5 & 1.9 & $34 \%$ & $11 \%$ & 7.7 & 6.2 & 0.6 & $10 \%$ & $19 \%$ \\
\hline Ho & 1.1 & 1.05 & 0.23 & $22 \%$ & $4 \%$ & 1.7 & 1.15 & 0.12 & $10 \%$ & $32 \%$ \\
\hline Er & 3.9 & 3.1 & 0.2 & $6 \%$ & $20 \%$ & 5.2 & 4.4 & 0.0 & $1 \%$ & $15 \%$ \\
\hline $\mathrm{Tm}$ & 0.73 & 0.76 & 0.26 & $34 \%$ & $-4 \%$ & 0.86 & 0.69 & 0.19 & $27 \%$ & $20 \%$ \\
\hline $\mathrm{Yb}$ & 4.6 & 3.5 & 0.1 & $3 \%$ & $\mathbf{2 5} \%$ & 5.4 & 4.5 & 0.2 & $4 \%$ & $16 \%$ \\
\hline Lu & 0.68 & 0.67 & 0.15 & $22 \%$ & $2 \%$ & 0.92 & 0.68 & 0.18 & $26 \%$ & $\mathbf{2 6} \%$ \\
\hline $\mathrm{Hf}$ & 4.7 & 3.8 & 1.4 & $38 \%$ & $19 \%$ & 5.2 & 3.4 & 1.0 & $28 \%$ & $34 \%$ \\
\hline
\end{tabular}

GSJ88: recomm ended value of Ando et al. (1989). Concentrations for major elements are calculated from the oxide forms. SIMS: quantitative SIMS value determined by empirical relationships shown in Fig. 1. Si concentration is internal standard. sigma, CV\%: standard derivation and relative error for reproduction on several independent runs, respectively. SIMS, sigma and CV\% were calculated from the mean secondary ion intensity ratios of each runs.

R.E.\%: relative error between GSJ88 and SIMS values. The errors over $20 \%$ are shown in bold face. 


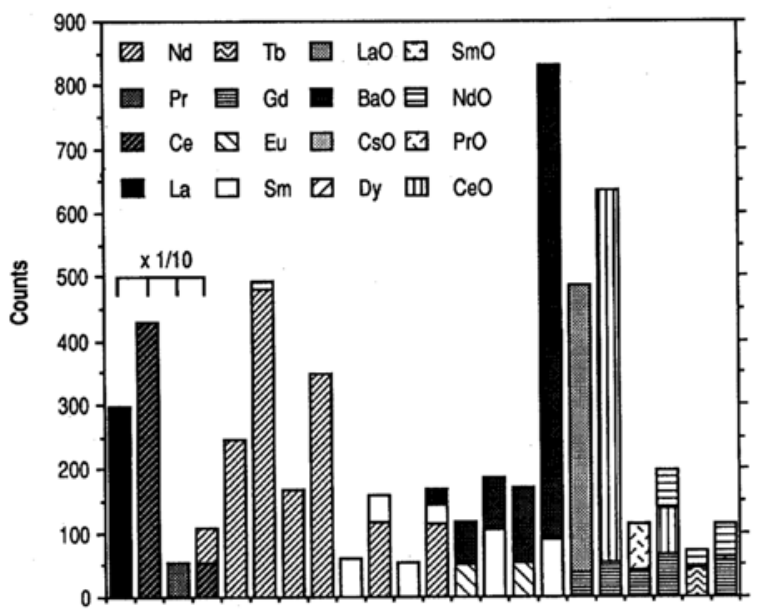

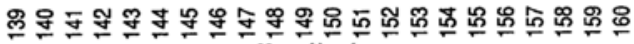
Mass Number

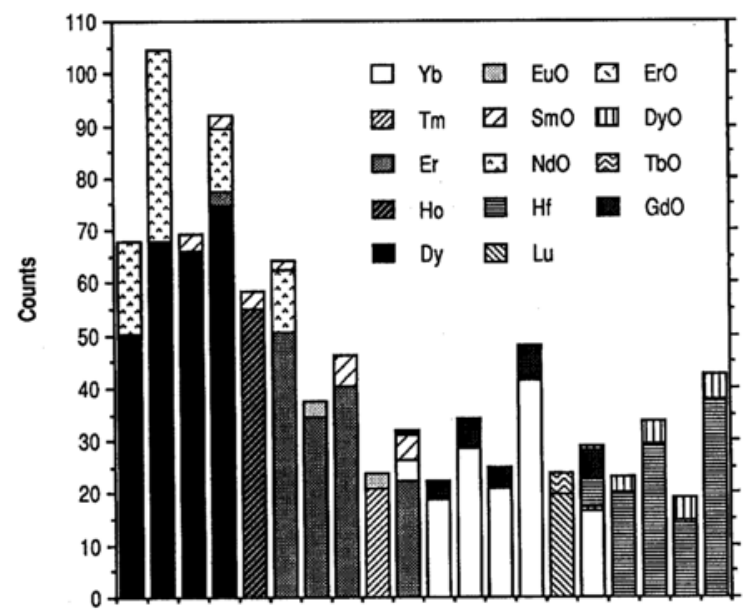

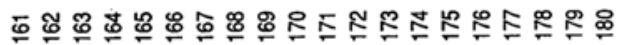

Mass Number

Fig. 2. Positive secondary ion mass spectra of JB-la glass standards. The spectra are deconvoluted into contributions from REE and their monoxide.

0.972 for $\mathrm{P}$ and $\mathrm{Sc}$, respectively.

\section{Rare Earth Elements and $\mathrm{Hf}$}

Energy filtering effectively removed all complex molecular ions interfering with the elements measured here. The only serious interferences on REE's were monoxide ions and other interferences were negligible as described by Zinner and Crozaz (1986). The contribution of oxide interferences under the energy filtering are shown in Fig. 2. The mass spectra were decon- voluted by the similar procedure reported by Zinner and Crozaz (1986).

For the quantitative analysis of REE's, we selected 22 masses only slightly affected by interferences. The masses are listed in Table 2. Using information on natural isotopic abundances, the contributions of interference were deconvoluted by the solution of simultaneous linearequations on these masses. It is difficult to calculate the contributions of $\mathrm{GdO}$ and $\mathrm{TbO}$ because of the low intensity of REE and the reason reported by Zinner and Crozaz (1986). Since the empirical relationship of the yields of REE monoxide ions (Reed, 1983) were also observed for the glass standards, the contributions of GdO and $\mathrm{TbO}$ on ${ }^{172} \mathrm{Yb},{ }^{174} \mathrm{Yb}$ and ${ }^{175} \mathrm{Lu}$, respectively, were subtracted by using the $\mathrm{NdO}^{+} / \mathrm{Nd}^{+}$ratio at the same measurement point. Similarly the intensity of $\mathrm{Hf}$ is obtained by subtracting the DyO contribution which is derived from the $\mathrm{SmO}^{+} / \mathrm{Sm}^{+}$ratio.

The results are plotted against the recommended values in Fig. 1. Since the JR-series data systematically deviate from the other glass standard trends, the plots except for JR-series were used for least-squares regression. All calibration curves are well defined linearly and pass through the origin within the random error of analysis.

Chondrite-normalized REE abundances determined by SIMS are compared with recommended values in Fig. 3. It can be seen that the match of both patterns is excellent for all glass standards, especially for light REE's. The error bars are dependent on the degrees of interference contributed and ion intensities of the masses (cf. Table 2 and Fig. 2).

Systematic shifts are clearly observed on the JR-series pattern (Fig. 3). The shifting may represent reduced REE ion yields caused by the $\mathrm{SiO}_{2}$ contents in the sample.

\section{Mass 64}

Of the five $\mathrm{Zn}$ isotopes, ${ }^{64} \mathrm{Zn}$ is the most abundant isotope $(48.6 \%)$. However, the secondary ion intensities of mass 64 show excellent correlation with $\mathrm{Ti}$ concentration (Fig. 1). The results show that the greatest species of mass 64 is $\mathrm{TiO}^{+}$ 
H. Yurimoto et al.

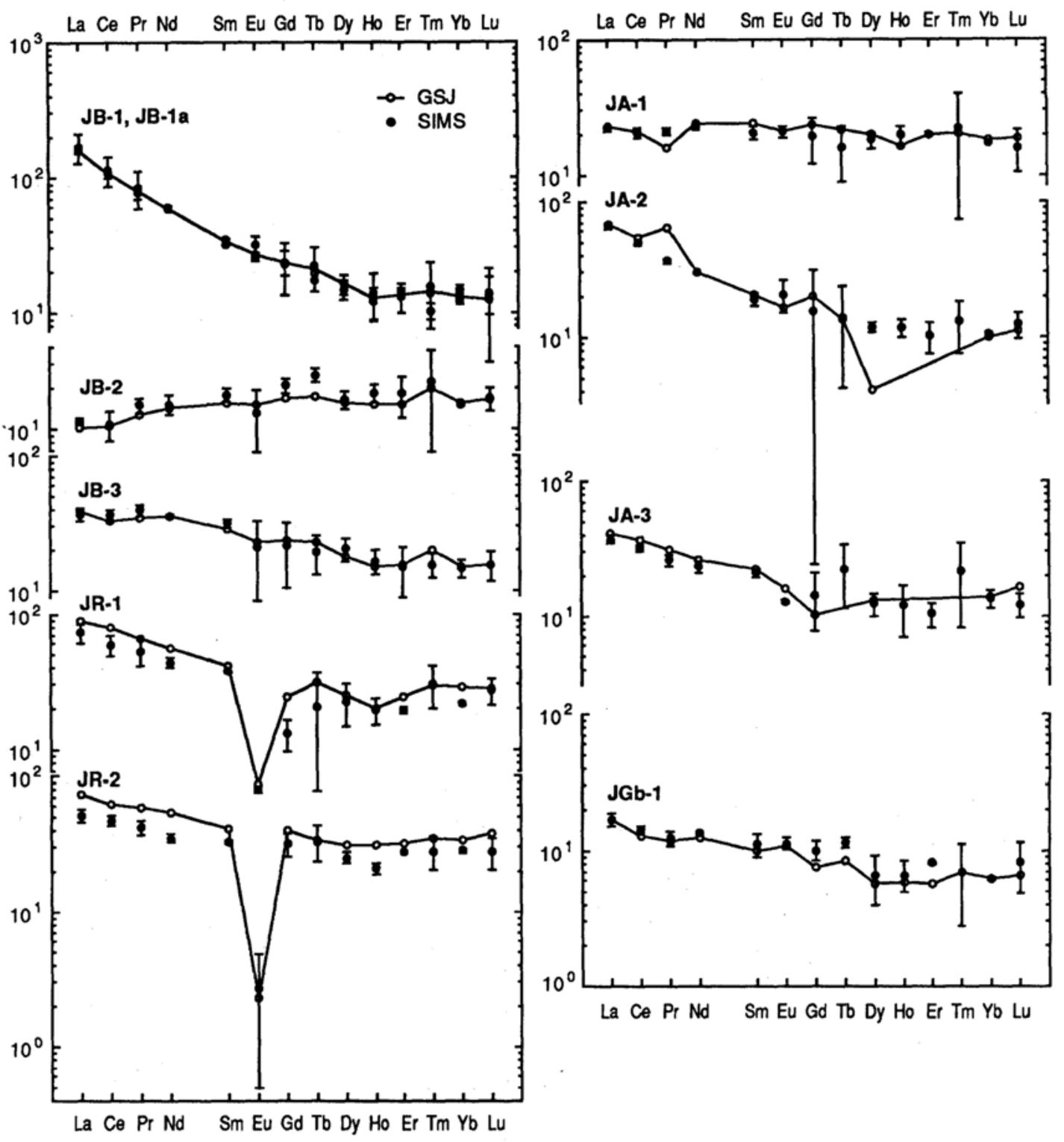

Fig. 3. Chondrite-normalized REE abundances in GSJ rock references against atomic number. The error bars represent the sigma column given in Table 1. REE values by Anders and Ebihara (1982) are used for chondritenormalization.

and the energy filtering is powerless in this case. dances.

This interference can be removed by the high mass resolution technique (Yurimoto and Sueno, 1984).

For $\mathrm{Zn}$ analysis using energy filtering, it is necessary to use the other isotope such as ${ }^{66} \mathrm{Zn}$ (27.9\%) or ${ }^{68} \mathrm{Zn}(18.8 \%)$. The isotopes, ${ }^{67} \mathrm{Zn}$ and ${ }^{70} \mathrm{Zn}$, may not be acceptable due to low abun-

SUMMARY
Glasses prepared from JA-1, JA-2, JA-3, JB-
1, JB-1a, JB-2, JB-3, JGb-1, JR-1 and JR-2 rock
standards were found to be homogeneous stan-
dards appropriate for SIMS micro-analysis.


Table 2. Elemental and interference species and the mass numbers

\begin{tabular}{cccc}
\hline Species & Masss Number & Species & Mass Number \\
\hline $\mathrm{Li}$ & 7 & $\mathrm{Y}$ & 89 \\
$\mathrm{Be}$ & 9 & $\mathrm{Zr}$ & 90 \\
$\mathrm{~B}$ & 11 & $\mathrm{Cs}$ & 133 \\
$\mathrm{Na}$ & 23 & $\mathrm{Ba}$ & 138 \\
$\mathrm{Mg}$ & 24,26 & $\mathrm{La}$ & 139 \\
$\mathrm{Al}$ & 27 & $\mathrm{Ce}$ & 140 \\
$\mathrm{Si}$ & 28,30 & $\mathrm{Pr}$ & 141 \\
$\mathrm{P}, \mathrm{Nd}$ & 31 & $\mathrm{Sm}$ & 143,146 \\
$\mathrm{~K}$ & 39 & $\mathrm{Eu}, \mathrm{BaO}$ & 147,149 \\
$\mathrm{Ca}$ & 40 & Gd, Dy, NdO, SmO & 151,153 \\
$\mathrm{Sc}, \mathrm{SiO}, \mathrm{AlO}$ & 45 & $\mathrm{~Tb}, \mathrm{NdO}$ & 160 \\
$\mathrm{Ti}$ & 47 & $\mathrm{Dy}, \mathrm{NdO}$ & 159 \\
$\mathrm{~V}$ & 51 & $\mathrm{Dy}, \mathrm{SmO}$ & 161,162 \\
$\mathrm{Cr}$ & 52 & $\mathrm{Ho}, \mathrm{SmO}$ & 163 \\
$\mathrm{Mn}$ & 55 & $\mathrm{Er}, \mathrm{NdO}, \mathrm{SmO}$ & 165 \\
$\mathrm{Fe}$ & $\mathrm{Er}, \mathrm{EuO}$ & 166,168 \\
$\mathrm{Co}$ & 56 & Tm, EuO & 167 \\
$\mathrm{Cu}$ & 59 & Yb, GdO & 169 \\
$\mathrm{TiO}$ & 63 & $\mathrm{Lu}, \mathrm{TbO}$ & 172,174 \\
$\mathrm{Rb}$ & 64 & $\mathrm{Hf}, \mathrm{DyO}$ & 175 \\
$\mathrm{Sr}$ & 85 & & $177,178,179,180$ \\
\hline
\end{tabular}

Simultaneous micro-analysis of major, minor and trace elements in ng samples is made possible by use of the glass standards. This method uses energy filtering and, for REE's and Hf, the contribution of simple oxide ions were corrected. Using specific SIMS operating conditions, linear calibration curves have been established for 38 elements $(\mathrm{Li}, \mathrm{Be}, \mathrm{B}, \mathrm{Na}, \mathrm{Mg}$, $\mathrm{Al}, \mathrm{P}, \mathrm{K}, \mathrm{Ca}, \mathrm{Sc}, \mathrm{Ti}, \mathrm{V}, \mathrm{Cr}, \mathrm{Mn}, \mathrm{Fe}, \mathrm{Co}, \mathrm{Cu}, \mathrm{Rb}$, $\mathrm{Sr}, \mathrm{Y}, \mathrm{Zr}, \mathrm{Cs}, \mathrm{Ba}, \mathrm{La}, \mathrm{Ce}, \mathrm{Pr}, \mathrm{Nd}, \mathrm{Sm}, \mathrm{Eu}, \mathrm{Gd}$, $\mathrm{Tb}, \mathrm{Dy}, \mathrm{Ho}, \mathrm{Er}, \mathrm{Tm}, \mathrm{Yb}, \mathrm{Lu}$ and $\mathrm{Hf}$ ). The typical accuracy of the analyses are within $10 \%$ of the recommended values for the elements over $1 \mathrm{ppm}$.

Complications were encountered as described below:

(1) Secondary ion yield for $\mathrm{Na}$ was enhanced in JF-, JG- and JR-series glasses which have relatively higher $\mathrm{Si}$ concentrations than the other standard glasses, whereas the yield of REE was reduced on JR-series glasses. The results demonstrate that systematic errors occur among silicates with different $\mathrm{Si}$ abundances for these elements.

(2) Energy filtering was ineffective in removing interferences on the $\mathrm{P}$ and Sc peaks. The detection limits of these elements are controlled by the background level of the masses.

Despite these problems, the empirical relationships obtained in this study are still useful for the application of quantitative SIMS to geological samples.

Acknowledgments-The authors are grateful to the late N. Onuma and A. Ando for invaluable advice and encouragement; I. Sakaguchi and K. Sato for assistance with SIMS analysis; H. Nagasawa, J. J. Papike and K. C. Galbreath for reading the manuscript. Partial support for this study was derived from the Grant-in-Aid for Scientific Research from the Ministry of Education of Japan.

\section{REFERENCES}

Anders, E. and Ebihara, M. (1982) Solar-system abundances of the elements. Geochim. Cosmochim. Acta 46, 2363-2380.

Ando, A., Kamioka, H., Terashima, S. and Itoh, S. (1989) 1988 values for GSJ rock reference samples, “Igneous rock series". Geochem. J. 23, 143-148.

Metson, J. B., Bancroft, G. M., McIntyre, N. S. and Chauvin, W. J. (1983) Suppression of molecular ions in the secondary ion mass spectra of minerals. Surf. Interface Anal. 5, 181-185. 
Nesbitt, H. W., Metson, J. B. and Bancroft, G. M. (1986) Quantitative major- and trace-element whole-rock analysis by secondary-ion mass spectrometry using the specimen isolation technique. Chem. Geol. 55, 139-160.

Ray, G. and Hart, S. R. (1982) Quantitative analysis of silicates by ion microprobe. Int. J. Mass Spec. Ion Phys. 44, 231-255.

Reed, S. J. B. (1983) Secondary-ion yields of rare earths. Int. J. Mass Spec. Ion Process. 54, 31-40.

Reed, S. J. B. (1984) Ion mass spectrometry ion probe analysis for rare earths. Scanning Electron Microsc. 11, 529-535.

Reed, S. J. B. (1986) Ion microprobe determination of rare earth elements in accessory minerals. Mineral. Mag. 50, 3-15.

Shimizu, N. (1986) Silicon-induced enhancement in secondary ion emission from silicates. Int. J. Mass Spec. Ion Process. 69, 325-338.

Shimizu, N. and Hart, S. R. (1982) Applications of the ion microprobe to geochemistry and cosmochemistry. Ann. Rev. Earth Planet. Sci. 10, 483-526.

Shimizu, N., Semet, M. P. and Allègre, C. J. (1978) Geochemical applications of quantitative ionmicroprobe analysis. Geochim. Cosmochim. Acta
42, 1321-1334.

Steele, I. M., Hervig, R. L., Hatcheon, I. D. and Smith, J. V. (1981) Ion microprobe techniques and analyses of olivine and low-Ca pyroxene. Am. Mineral. 66, 526-546.

Williams, P. (1985) Secondary ion mass spectrometry. Ann. Rev. Mater. Sci. 15, 517-548.

Yurimoto, H., Kurosawa, M. and Sueno, S. (1989) Hydrogen analysis in quartz crystals and quartz glasses by secondary ion mass spectrometry. Geochim. Cosmochim. Acta 53, 751-755.

Yurimoto, H. and Sueno, S.(1984) Anion and cation partitioning between olivine, plagioclase phenocrysts and the host magma: A new application of ion microprobe study. Geochem. J. 18, 8594.

Yurimoto, H. and Sueno, S. (1985) Quantitative analysis of silicate glasses by SIMS. Ann. Meet. Abs. Geochem. Soc. Jpn. 1985, $2 \mathrm{D} 10$.

Yurimoto, H. and Sueno, S. (1987) Secondary ion mass spectrometry for insulators. J. Crystall. Soc. Jpn. 29, 259-269.

Zinner, E. and Crozaz, G. (1986) A method for the quantitative measurement of rare earth elements in the ion microprobe. Int. J. Mass Spec. Ion Process. 69, 17-18. 